\title{
Thyroid
}

\section{Feasibility of Intraoperative Neuromonitoring During Thyroid Surgery using Trans-cartilage Surface Recording electrodes.}

\begin{tabular}{|c|c|}
\hline Journal: & Thyroid \\
\hline Manuscript ID & THY-2017-0680.R1 \\
\hline Manuscript Type: & Invited Paper \\
\hline Date Submitted by the Author: & $\mathrm{n} / \mathrm{a}$ \\
\hline Complete List of Authors: & $\begin{array}{l}\text { Wu, Che-Wei; Department of Otolaryngology, Kaohsiung Medical University } \\
\text { Hospital, Kaohsiung Medical University, Kaohsiung City - Taiwan, } \\
\text { Chiang, Feng-Yu; Department of Otolaryngology, Kaohsiung Medical } \\
\text { University Hospital, Kaohsiung Medical University, Kaohsiung City - Taiwan } \\
\text { Randolph, Gregory; Massachusetts Eye and Ear Infirmary, , Boston, MA, } \\
\text { Otolaryngology } \\
\text { Dionigi, Gianlorenzo; Universita degli Studi di Messina, Human Pathology } \\
\text { Kim, Hoon Yub; Korea University, Department of Surgery, Division of } \\
\text { Breast and Endocrine Surgery, Minimally Invasive Surgery and Robotic } \\
\text { Surgery Center, KUMC Thyroid Center Korea University, Anam Hospital } \\
\text { Seoul, Korea } \\
\text { Lin, Yi-Chu ; Kaohsiung Medical University, Otorhinolaryngology } \\
\text { Chen, Hui-Chun; Kaohsiung Medical University, Nursing } \\
\text { Chen, Hsiu-Ya ; Kaohsiung Medical University } \\
\text { kamani, dipti; Massachusetts Eye and ear Infirmary, Div of Thyroid and } \\
\text { Parathyroid Surgery, dept of Otolaryngology } \\
\text { Tsai, Tsung-Yi; Kaohsiung Medical University, Otolaryngology-Head and } \\
\text { Neck Surgery } \\
\text { Lu, I-Cheng; Kaohsiung Medical University, Anesthesiology } \\
\text { Chang, Pi-Ying; Kaohsiung Medical University, Anesthesiology }\end{array}$ \\
\hline Keyword: & $\begin{array}{l}\text { Surgery-Adult Thyroid, ENT, Surgery-Adult Thyroid, General, Animal } \\
\text { Physiology, Clinical Research }\end{array}$ \\
\hline \multirow[t]{2}{*}{$\begin{array}{r}\text { Manuscript Keywords (Search } \\
\text { Terms): }\end{array}$} & $\begin{array}{l}\text { Intraoperative Neuromonitoring, Thyroid Surgery, Recurrent laryngeal } \\
\text { nerve, Transcartilage Recording, Electromyography, Surface electrode }\end{array}$ \\
\hline & $\begin{array}{l}\text { Background: } \\
\text { Intraoperative neural monitoring (IONM) has gained widespread } \\
\text { acceptance as an adjunct to the gold standard of visual identification of the } \\
\text { recurrent laryngeal nerve (RLN) during thyroid surgery. Currently, } \\
\text { laryngeal electromyography (EMG) recording during IONM is almost always } \\
\text { performed using endotracheal tube (ETT) surface electrodes placed } \\
\text { adjacent to vocal folds originating from the inner surface of the thyroid } \\
\text { cartilage (TC). Therefore, we hypothesized that surface recording } \\
\text { electrodes placed on the outer surface of the TC should enable access to } \\
\text { the EMG response of the vocal folds during IONM. The aims of this }\end{array}$ \\
\hline
\end{tabular}


experimental study were to evaluate the feasibility of the transcartilage approach for laryngeal EMG recording during IONM.

Methods:

A porcine model (12 pigs and 24 RLN sides) with well-established applicability in IONM research was used for the experiments. Both ETT electrodes adjacent to vocal folds and adhesive pre-gelled electrodes on the TC were used for EMG recording during IONM. Electrically evoked EMG signals detected by both electrode types were recorded and analyzed. EMG changes during tracheal displacement and RLN traction injury were compared.

Results:

Both the ETT and TC electrodes recorded typical laryngeal EMG waveforms evoked by a $1 \mathrm{~mA}$ stimulus current applied on both sides of the RLNs and vagus nerves (VNs). Under RLN stimulation, the mean EMG amplitudes recorded with the ETT and TC electrodes were $973 \mu \mathrm{V}( \pm 179)$ and $695 \mu \mathrm{V}$ $( \pm 150)$, respectively. Under $V N$ stimulation, the mean amplitudes were $841 \mu \mathrm{V}( \pm 163)$ and $607 \mu \mathrm{V}( \pm 162)$, respectively. When upward displacement of the trachea was experimentally induced, the TC electrodes showed less variation in recorded EMG signals compared to ETT electrodes. When RLN traction stress was experimentally induced, both the ETT and TC electrodes accurately recorded the typical EMG pattern of progressively degrading amplitude and gradual recovery after release of traction.

Conclusions:

This study confirmed the feasibility of using transcartilage surface electrodes for recording of laryngeal EMG signals evoked during IONM in an animal model. Before practical application of this approach in clinical thyroid surgery, however, further studies are needed to improve electrode designs by optimizing their shapes and sizes, and increasing their adhesive stability and sensitivity. 


\section{Title Page}

2 Title: Feasibility and Stability of Intraoperative Neuromonitoring During Thyroid

Surgery Using Non-invasive Adhesive Trans-Cartilage _ Surface Recording Electrodes-

4 Article Category: Original Articles

5 Authors: Che-Wei Wu, MD, $\mathrm{PhD}^{1} \underline{\text { cwwu@kmu.edu.tw; }}$

$6 \quad$ Feng-Yu Chiang, $\mathrm{MD}^{1}$ fychiang@kmu.edu.tw;

7 Gregory W. Randolph, MD, FACS, FACE ${ }^{2,3}$ gregory_randolph@meei.harvard.edu;

8 Gianlorenzo Dionigi, MD, FACS ${ }^{4}$ gdionigi@unime.it;

9 Hoon Yub Kim, MD, PhD ${ }^{5}$ hoonyubkim@gmail.com;

10 Yi-Chu Lin, MD, MMS ${ }^{1}$ reddust0113@yahoo.com.tw;

11 Hui-Chun Chen, $\mathrm{RN}^{6}$ s334@ms19.hinet.net;

12 Hsiu-Ya Chen, NA ${ }^{7}$ liga888@yahoo.com.tw;

13 Dipti Kamani, MD ${ }^{2,3}$ dipti_kamani@meei.harvard.edu;

14 Tsung-Yi Tsai, MD $1000448 @$,kmuh.org.tw;

15 I-Cheng Lu, MD, $\mathrm{PhD}^{7}$ u9251112@gmail.com;

16 Pi-Ying Chang, MD ${ }^{7 *}$ annabelle69@gmail.com;

\section{Affiliations}

18 1. Department of Otolaryngology-Head and Neck Surgery, Kaohsiung Municipal

19 Hsiao-Kang Hospital, Kaohsiung Medical University Hospital, Faculty of Medicine, 
1 College of Medicine, Kaohsiung Medical University, Kaohsiung, Taiwan.

2 2. Division of Thyroid and Parathyroid Endocrine Surgery, Department of

3 Otolaryngology, Massachusetts Eye and Ear Infirmary; Department of Otology and

4 Laryngology, Harvard Medical School, Boston, Massachusetts, U.S.A.

5 3. Division of Surgical Oncology, Department of Surgery, Massachusetts General

6 Hospital, Harvard Medical School Boston, MA

7 4. Division for Endocrine Surgery, Department of Human Pathology in Adulthood and

8 Child-hood "G. Barresi", University Hospital G. Martino, University of Messina, Via

9 C. Valeria 1, 98125, Messina, Sicily, Italy

10 5. Department of Surgery, Korea University College of Medicine, Seoul, Korea.

11 6. Department of Nursing, Kaohsiung Medical University Hospital, Kaohsiung

12 Medical University, Kaohsiung, Taiwan.

13 7. Department of Anesthesiology, Kaohsiung Municipal Ta-Tung Hospital, Kaohsiung

14 Municipal Hsiao-Kang Hospital, Kaohsiung Medical University Hospital, Faculty of

15 Medicine, College of Medicine, Kaohsiung Medical University, Kaohsiung, Taiwan.

16 Running Title: Transcartilage RLN monitoring in Thyroid Surgery

17 Key Words: Intraoperative Neuromonitoring, Thyroid Surgery, Recurrent laryngeal

18 nerve, Transcartilage Recording, Electromyography, Surface electrode 19 


\section{ABSTRACT:}

\section{Background:}

3 Intraoperative neural monitoring (IONM) has gained widespread acceptance as an

4 adjunct to the gold standard of visual identification of the recurrent laryngeal nerve

5 (RLN) during thyroid surgery. Currently, laryngeal electromyography (EMG)

6 recording during IONM is almost always performed using endotracheal tube (ETT)

7 surface electrodes placed adjacent to vocal folds originating from the inner surface of

8 the thyroid cartilage (TC). Therefore, we hypothesized that surface recording

9 electrodes placed on the outer surface of the TC should enable access to the EMG

10

11

12

13

14

15 Methods:

16

17

18

19

response of the vocal folds during IONM-and should obtain more stable signals during surgical manipulation compared to conventional ETT electrodes. The aims of

this experimental study were to evaluate the feasibility of the transcartilage approach

for laryngeal EMG recording during IONM. stability of IONM using non-invasive

adhesive trans-cartilage recording electrodes 
1 evoked EMG signals detected by both electrode types were recorded and analyzed. were compared.

\section{Results:}

5 Both the ETT and TC recording electrodes recorded typical laryngeal EMG

6 waveforms evoked by a $1 \mathrm{~mA}$ stimulus current applied on both sides of the RLNs and

7 vagus nerves (VNs). Under RLN stimulation, the mean EMG amplitudes recorded

8

9

10

\section{Conclusions: \\ 17 \\ 18 \\ 19 \\ This study confirmed the feasibility of using transcartilage surface electrodes for recording of laryngeal EMG signals evoked during IONM in an animal model. For monitoring RLN function during thyroid surgery, TC electrodes provide a simple and} with the ETT and TC electrodes were $973991 \mu \mathrm{V}( \pm-\underline{179190)}$ and $695658 \mu \mathrm{V}( \pm$ $\underline{150132)}$, respectively. Under VN stimulation, the mean amplitudes were $\underline{841} 842 \mu \mathrm{V}$ $( \pm 161 \underline{163})$ and $563 \underline{607} \mu \mathrm{V}( \pm 134 \underline{162})$, respectively. When upward displacement of the trachea was experimentally induced, the TC electrodes showed less variation in recorded EMG signals compared to ETT electrodes. When RLN traction stress was experimentally induced, both the ETT and TC electrodes accurately recorded the typical EMG pattern of progressively degrading amplitude and gradual recovery after release of traction. 
effective alternative to ETT electrodes Before practical application of this approach in

2

clinical thyroid surgery, however, further studies are needed to improve electrode

3

designs by optimizing their shapes and sizes, and increasing their adhesive stability and sensitivity.

5

6 


\section{MAIN BODY}

\section{Introduction}

3 Although thyroidectomy is now a commonly performed procedure worldwide,

4 postoperative vocal cord palsy is still common. In patients undergoing

5 thyroidectomy, the average incidence of temporary vocal cord palsy is $9.8 \%$, and the

6 average incidence of permanent vocal cord palsy is $2.3 \% .(1,2)$ For identification of

7 the recurrent laryngeal nerve (RLN) during thyroid surgery, the use of intraoperative

8 neural monitoring (IONM) has gained widespread acceptance as an adjunct to the

9 gold standard of visual identification of the RLN.(3) Additionally, IONM can

10 facilitate functional preservation of the RLN and the external branch of the superior

11 laryngeal nerve, (4) detect anatomic variations(5-7), elucidate nerve injury

12 mechanisms, $(8,9)$ and enable intraoperative predictions of postoperative vocal cord

13 function and its implications in patients undergoing a planned bilateral surgery .(3, 10 , $14 \quad 11)$

15 Currently, monitoring systems that use endotracheal tube (ETT) surface electrodes

16 are the most widely used commercially available monitoring systems.(3) These

17 ETT-based systems record electromyographic (EMG) data from the vocal folds (i.e.,

18 thyroarytenoid or vocalis muscles). During IONM, a handheld stimulation probe is

19 used to evoke the laryngeal EMG response by depolarizing the RLN or vagus nerve 
1 (VN). The EMG response is typically quantified by both the amplitude and latency of

2 the signal obtained during RLN or VN stimulation. The EMG amplitude may

3 correlate with the number of depolarized muscle fibers, which then affects RLN

4 function. Latency is related to the time of the conducted action potential and

5 response.(12-14) The main limitation of ETT-based systems is that a high-quality

6 recording requires constant contact between the electrodes and vocal folds during

7 surgery.(3, 13, 15-18) A malpositioned or displaced ETT can cause false IONM

8 signals $(8,11,13,15-17,19)$ resulting in the need for intraoperative verification or

9 readjustment of the ETT position, which can be complicated and time-consuming.

10 Since the vocal fold originates from the inner surface of the thyroid cartilage (TC)

11 (Fig. 1A), we hypothesized that surface recording electrodes placed on the outer

12 surface of the TC should obtain recordings of the EMG response of the vocal fold.

13 We also hypothesized that, compared to ETT electrodes, the TC electrodes would

14 obtain less affected EMG signals during tracheal movement and would detect similar

15 adverse signals caused by RLN injury. Thus, this study reviewed a novel

16 transcartilage approach in which non-invasive adhesive surface electrodes were

17 attached to the outer surface of the TC (Fig. 1A-B) for recording EMG signals during

18 IONM. Experiments were performed to evaluate the feasibility and stability of the

19 proposed approach and to compare its signal stability and accuracy with that of 


\section{Materials and Methods}

3 Experimental animals, anesthesia, and ETT surface electrodes

4 Twelve Ten male pigs (age, 3-4 months; weight, 20-24-25 kg) obtained from the

5 Laboratory Animal Center of Kaohsiung Medical University, Taiwan were studied.

6 The study protocol was approved by the Institutional Animal Care and Use

7 Committee of Kaohsiung Medical University. All experiments were performed

8 according to institutional guidelines that comply with national and international

9 regulations for animal experiments_. including the $3 \mathrm{R}$ principles (replacement, reduction and refinement). The porcine model used for the experiments in this study is

11 well-established in RLN and IONM research, and the EMG parameters (amplitude,

12 latency, and threshold) of the model are consistent with human data.(12, 20-22)

13 Each animal was premedicated with intramuscular azaperone $(4 \mathrm{mg} / \mathrm{kg}) 30 \mathrm{~min}$

14 before the experiment. With the animal in a prone position, general anesthesia was

15 induced by inhalation of $2-4 \%$ sevoflurane in oxygen administered from a plastic

16 bottle. A bolus of propofol $1 \mathrm{mg} / \mathrm{kg}$ was administered intravenously with a 24-gauge

17 peripheral intravenous catheter placed on the ear. The pig was then intubated with

18 an EMG ETT (TriVantage EMG tube, size 6 ID; Medtronic, Jacksonville, FL) (Fig.

19 1C). The proper EMG tube position was verified and confirmed by the 
1 anesthesiologist. After positional stabilization of the tube, the pig was placed in the

2 supine position with the neck extended (Fig. 2A). Anesthesia was maintained with

4 tidal volume of $8-12 \mathrm{ml} / \mathrm{kg}$, and the respiratory rate was set to $12-14$ breaths $/ \mathrm{min}$.

5 Body temperature was maintained with a circulating water mattress maintained at $6 \quad 40^{\circ} \mathrm{C}$.

7

8 Operation, TC surface electrodes, and experimental design

9 A long, transverse neck skin incision was made, and subplatysmal flaps were

10 raised. The neck was exposed with a self-retaining thyroidectomy retractor

11 (WIDOX ${ }^{\circledR}$, MOSS Spa, Lesa, Italy). A pair of adhesive pre-gelled electrodes

12 (Neotrode II ${ }^{2}$, ConMed Corporation, UTICA, NY) were attached to the left and right

13 lamina of the TC (Fig. 2B). The channel leads from the EMG ETT and TC

14 electrodes were connected to the monitoring system (NIM-Response 3.0, Medtronic).

15 The monitoring system generated stimuli with a time window set to $50 \mathrm{~ms}$ and an

16 amplitude scale set to $0.2 \mathrm{mV} /$ division. The pulsed stimuli were $100 \mu \mathrm{s}$ in duration

17 and $4 \mathrm{~Hz}$ in frequency. The event capture was activated at a threshold of $100 \mu \mathrm{V}$.

18 In each pig $(\mathrm{n}=10 \underline{12})$, bilateral RLNs $(\mathrm{n}=20 \underline{24})$ and VNs $(\mathrm{n}=20 \underline{24})$ were 19 localized, identified, and carefully exposed with a handheld stimulation probe 
1 (Ball-tip 1mm, Medtronic) (Fig. 2B). Each nerve was stimulated with a $1 \mathrm{~mA}$

2 current, and the

3 waveform morphologies of the electrically evoked EMG signals detected by both the

4 ETT and TC electrodes were displayed on the monitor (Figs. 2C-D). Baseline

5 amplitudes and latencies of EMG responses were recorded for further analysis.

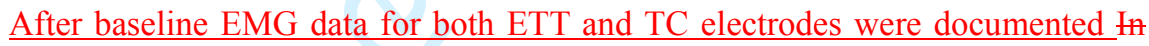
each pig $(n-10)$, the stability of EMG signals recorded by both electrode types during surgical manipulation was evaluated and compared under experimentally induced tracheal displacement in each pig (Figs. 3A-B). In four animals, the accuracy of the ETT and TC electrodes in reflecting adverse EMG changes of stressed RLN was further evaluated and compared under experimentally induced RLN traction during continuous IONM (Figs. 3C-D), tracheal displacement induced by slowly pushing the cricoid cartilage $2 \mathrm{~cm}$ upward during continuous left $\mathrm{VN}$ stimulation with an automatic periodic stimulating electrode (APS; Medtronic) (Fig. 3A,B). Such cricoid displacement mimicked thyroid greater tracheal and laryngeal retraction at surgery.

\section{$\underline{\text { Statistical Analysis }}$}

Recorded EMG data were expressed as mean with standard deviation. Mean EMG amplitudes and mean EMG latencies for each stimulation site were compared using paired T-tests. All p-values were two-tailed, and a $\mathrm{P}$ value less than 0.05 was 


\section{Results} and 20-24 RLNs). In all animals, both the ETT and TC electrodes successfully

7 recorded typical evoked laryngeal EMG waveforms from the RLNs and VNs under

8 stimulation with $1 \mathrm{~mA}$ (Figs. 2C-D.).

Figure 4 compares the Table 1 shows the evoked laryngeal EMG amplitudes and latencies recorded from the ETT and TC electrodes. In each group, the ETT electrodes recorded significantly higher EMG amplitudes compared to the TC electrodes $(\mathrm{P}<$ 0.05). However, ETT electrodes and TC electrodes did not significantly differ in recorded EMG latency data $(\mathrm{P}>0.05)$. Mean EMG amplitudes recorded under RLN stimulation were $991 \underline{973} \mu \mathrm{V}( \pm 190 \underline{179})$ for ETT electrodes and $658 \underline{695} \mu \mathrm{V}( \pm$ 132150) for TC electrodes. Mean EMG amplitudes recorded under VN stimulation were $842 \underline{841} \mu \mathrm{V}( \pm 161 \underline{163})$ for ETT electrodes and $563-\underline{607} \mu \mathrm{V}( \pm 134 \underline{162})$ for TC electrodes. Mean EMG latencies recorded under RLN stimulation were $2.982 .96 \mathrm{~ms}$ $( \pm 0.14 \underline{0.15})$ for ETT electrodes and $3.03 \underline{3.01} \mathrm{~ms}( \pm 0.16 \underline{0.18})$ for TC electrodes. Mean EMG latencies recorded under right VN stimulation were $4.80 \underline{4.75} \mathrm{~ms}$ 
$( \pm 0.23 \underline{0.24})$ for ETT electrodes and $4.85 \underline{4.79} \mathrm{~ms}( \pm 0.27 \underline{0.28})$ for TC electrodes.

2
Mean EMG latencies recorded under left VN stimulation were $8.55 \underline{8.22} \mathrm{~ms}$ $( \pm 0.560 .72)$ for ETT electrodes and $8.58 \underline{8.25} \mathrm{~ms}( \pm 0.47 \underline{0.65})$ for TC electrodes.

\section{When upward cricoid/ tracheal displacement was experimentally induced, the EMG}

signals from ETT electrodes varied significantly. The mean changes in EMG-

amplitude recorded after upward displacement of $1 \mathrm{~cm}$ were $7.8 \pm 1.7 \%$ (range, 5 to-

11\%) for ETT electrodes and $45.6 \pm 8.2 \%$ (range, 28 to $53 \%$ ) for TC electrodes. The mean changes in EMG amplitude recorded after upward displacement of $2 \mathrm{~cm}$ were-

$44.3 \pm 4.6 \%$ (range, 8 to $25 \%$ ) for ETT electrodes and $68.4 \pm 10.6 \%$ (range, 53 to 85\%) for TC electrodes_ETT electrodes were $66 \pm 9.6 \%$ (range from 53 to $80 \%$ ). In contrast, upward tracheal displacement did not affect the EMG signals from TC

electrodes (with the exception of some artefactual responses to pushing force on theericoid and tracheal cartilage (Fig3C). Mean EMG amplitudes change at $2 \mathrm{~cm}$ displacement recorded by TC electrodes were $13 \pm 3.3 \%$ (range from 8 to $19 \%$ ).

The tracheal displacement study evaluated the real-time EMG signal changes

recorded by ETT and TC electrodes. The results showed that, in both electrode types, tracheal displacement affected the recorded EMG signals (Fig 5). The mean changes in EMG amplitude recorded after upward displacement of $1 \mathrm{~cm}$ were $7.8 \pm 1.7 \%$ (range, 5 to $11 \%$ ) for ETT electrodes and $45.6 \pm 8.2 \%$ (range, 28 to $53 \%$ ) for TC 
electrodes. The mean changes in EMG amplitude recorded after upward

displacement of $2 \mathrm{~cm}$ were $14.3 \pm 4.6 \%$ (range, 8 to $25 \%$ ) for ETT electrodes and

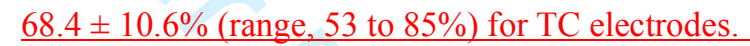

The RLN traction stress study evaluated the real-time EMG signal changes 


\section{Discussion}

2 We hypothesized that surface recording electrodes on the outer surface of the TC

electrodes would obtain more stable signals during tracheal movement compared to ETT electrodes. This hypothesis was tested in a porcine model with well-established

6 applicability in RLN and IONM research.(12, 20-22) The experiments confirmed that 
1 injury during thyroid surgery. $(23,24)$ The leading mechanism of RLN injury is

2 nonstructural functional nerve injury, not transection. $(8,9,25)$ Therefore, IONM is

4 to visual confirmation of RLN structural integrity.(3) In thyroid surgery, IONM is

5 almost always performed with commercially available ETT surface recording

6 electrodes placed adjacent to the vocal folds. For accurate monitoring, the ETT must

7 be the appropriate size and must be correctly aligned.(13, 18) The experiments in this study showed that, in contrast with the time-consuming and cumbersome procedures required to place ETT electrodes by anesthesiologists during intubation, the setup procedure for TC electrodes can be performed by surgeons in less than 1 minute, and the correct electrode locations can be confirmed by direct visualization.

12 In addition, intraoperative manipulation and traction of the thyroid lobe and trachea

13 can easily displace the contact between the ETT electrodes and vocal fold.

14 Correcting the resulting false IONM results may require the anesthesiologist to verify

15 and readjust the electrodes intraoperatively.(8, 11, 13, 15-17) Several studies have

16 investigated alternative recording approaches to address the limitations of ETT

17 electrodes. For example, Alon and Hinni(26) and Petro et al(27) described the use of

18 one or two paired needle electrodes inserted through the cricothyroid membrane and 19 into the thyroarytenoid muscles. Recently, Chiang et al(28) described the use of two 
1 single needle electrodes inserted into the sub-perichondrium of the TC for IONM of

2 the RLN. Needle electrode approaches theoretically overcome most limitations of

3 ETT electrodes but are not entirely non-invasive. Needle electrodes can still cause

4 laryngeal hematomas, lacerations, infections, or ruptured endotracheal cuffs.

5 Additionally, a needle electrode insertion may be difficult due to scar tissue in

6 revision surgery or due to calcified TC in elderly patients. The Massachusetts Eye and

7 Ear Infirmary group recently reported their animal experiments(29) and preliminary

8 clinical experience(30) in using postcricoid surface electrodes to obtain EMG

9 waveforms at the posterior cricoarytenoid (PCA) muscle. Although their experimental

10 results showed that postcricoid electrodes reliably recorded PCA EMG waveforms,

11 constant verification of contact between the postcricoid electrodes and PCA muscle

12 may be required because the postcricoid electrodes are susceptible to displacement 13 during surgical manipulation.

14 Ideally, the recording method used during IONM should be non-invasive, reliable,

15 reproducible, easy to perform, and cost-effective. Compared to ETT electrodes and

16 intra-muscular or intra-cartilage needle electrodes, an adhesive electrode placed on

17 the TC is ostensibly simpler, faster, and less invasive, i.e., it does not leave needle

18 tracts and should cause less trauma in the mucosa, cartilage, or muscles. Adhesive

19 electrodes are also less expensive. In our institution, for example, an EMG ETT or a 
1 paired needle electrode costs several hundred dollars more than an adhesive electrode.

.

children. Therefore, these electrode types are somewhat larger than necessary for

use on the TC. Recording traces can be distorted by the far field potentials from

multitude of muscles attached to the larynx and underlying the surface electrodes (e.g..

sterno-hyoid muscle, thyro-hyoid muscle, and inferior constrictor muscle). For

example, the experiments in this study showed that a stimulation current exceeding 10

$\underline{\mathrm{mA}}$ causes some shunt stimulation of the ansa cervicalis and/ or the strap muscles.

Evoked muscle potentials could be recorded more easily by large surface electrodes

on the TC. However, no obvious far field potentials were recorded under the standard

$\underline{\text { stimulation current of } 0.5-3 \mathrm{~mA} \text { (Figs. 1C-D). Therefore, new electrode designs with }}$ $\underline{\text { sizes and shapes optimized for TC recording are needed, and further animal and }}$ 
clinical studies are needed to evaluate their susceptibility to far field potentials.

Third, although this study showed that TC electrodes obtained more stable signals

than ETT electrodes did during upward displacement of the trachea, the adhesive

pre-gelled surface electrodes may still be dislodged during surgical manipulations.

Dislodgement can occur because the vector of manipulative force applied during

clinical thyroid surgery tends to include both rotatory force and displacement.

$\underline{\text { Rotatory force would likely displace the contact between the ETT electrodes and }}$

vocal fold and may also dislodge the TC surface electrodes. Although a dislodged TC

electrode can be confirmed by direct visualization and can be corrected immediately,

the troubleshooting algorithm for loss of signal must be modified to address this

limitation. To minimize dislodgement, the stability of future electrode designs must

be increased by improving their adhesion to the TC. Smaller electrodes are also

needed to minimize the possibility of damage to cartilage or nearby muscles upon

electrode removal.

This study has some limitations. First, this study used an animal model with a

moderate sample size. Although this model has proven useful and effective for

evaluating electrophysiologic correlates of laryngeal EMG during IONM in previous-

studies,(20-22) and although the results for the model were highly consistent with the-

twenty RLN or VN sides in the current study (Table 1), further studies in human 

subjects are needed. Second Finally, the mean EMG amplitude recorded with

2 adhesive electrodes on the outer surface of the TC was lower than that recorded with

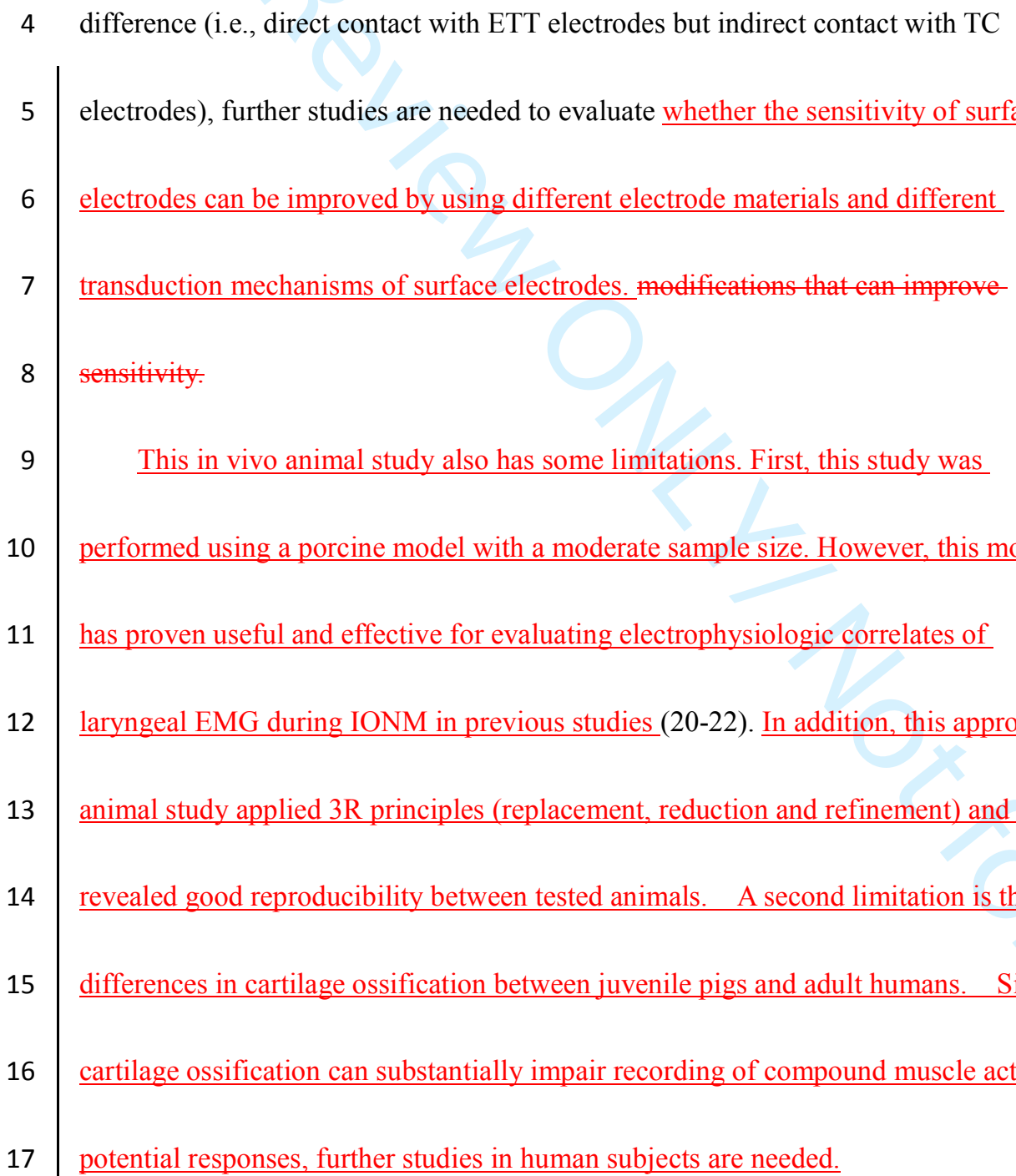


vocal fold during IONM of the RLN. A remaining challenge in the practical clinical

application of transcartilage IONM is development of surface electrodes that provide

a desirable combination of proper size/shape, high adhesive stability, and high

manipulation. This approach provides a simple and effective alternative to using an

EMG ETT to monitor RLN function during thyroid surgery.

8

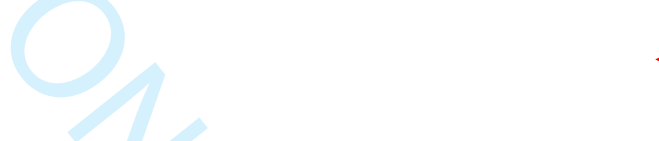

Formatted: Indent: First line: $0.33 "$, No widow/orphan control, Don't adjust space between Latin and Asian text, Don't adjust space between Asian text and numbers 


\section{Acknowledgments}

2 This study was oral presented at the 3rd World Congress on Thyroid Cancer, July

3 27-30, 2017 in Boston. The authors gratefully acknowledge the technical assistance

4 provided by Pao-Chu Hun (veterinarian, Laboratory Animal Center, KMU). This

5 study was supported by grants from the Kaohsiung Medical University Hospital,

6 Kaohsiung Medical University (KMUH106-6R49) and the Ministry of Science and

7 Technology, Taiwan (MOST 105-2314-B-037-010, 106-2314-B-037-042-MY2.)

8 Disclosure Statement

9 The authors have no conflict of interests to declare.

10

11 Name and address of corresponding author

12 Pi-Ying Chang, MD.

13 Department of Anesthesiology, Kaohsiung Municipal Ta-Tung Hospital, Kaohsiung

14 Medical University Hospital, Kaohsiung Medical University, Kaohsiung, Taiwan.

15100 TzYou First Road, Kaohsiung City 807, Taiwan.

16 E-mail address: annabelle69@gmail.com

17 


\section{References}

2

Jeannon JP, Orabi AA, Bruch GA, Abdalsalam HA, Simo R 2009 Diagnosis of recurrent laryngeal nerve palsy after thyroidectomy: a systematic review. International journal of clinical practice 63:624-629.

2. Haugen BR, Alexander EK, Bible KC, Doherty GM, Mandel SJ, Nikiforov YE, Pacini F, Randolph GW, Sawka AM, Schlumberger M, Schuff KG, Sherman SI, Sosa JA, Steward DL, Tuttle RM, Wartofsky L 20162015 American Thyroid Association Management Guidelines for Adult Patients with Thyroid Nodules and Differentiated Thyroid Cancer: The American Thyroid Association Guidelines Task Force on Thyroid Nodules and Differentiated Thyroid Cancer. Thyroid 26:1-133.

3. Randolph GW, Dralle $H$, Abdullah $H$, Barczynski $M$, Bellantone $R$, Brauckhoff $M$, Carnaille B, Cherenko $S$, Chiang FY, Dionigi G, Finck C, Hartl D, Kamani D, Lorenz K, Miccolli $P$, Mihai R, Miyauchi A, Orloff L, Perrier N, Poveda MD, Romanchishen A, Serpell J, Sitges-Serra A, Sloan T, Van Slycke S, Snyder S, Takami H, Volpi E, Woodson G 2011 Electrophysiologic recurrent laryngeal nerve monitoring during thyroid and parathyroid surgery: international standards guideline statement. The Laryngoscope 121 Suppl 1:S1-16.

4. Barczynski $M$, Randolph GW, Cernea $C R$, Dralle $H$, Dionigi $G$, Alesina PF, Mihai R, Finck C, Lombardi D, Hartl DM, Miyauchi A, Serpell J, Snyder S, Volpi E, Woodson G, Kraimps JL, Hisham AN 2013 External branch of the superior laryngeal nerve monitoring during thyroid and parathyroid surgery: International Neural Monitoring Study Group standards guideline statement. The Laryngoscope 123 Suppl 4:S1-14.

5. Chiang FY, Lu IC, Chen HC, Chen HY, Tsai CJ, Hsiao PJ, Lee KW, Wu CW 2010 Anatomical variations of recurrent laryngeal nerve during thyroid surgery: how to identify and handle the variations with intraoperative neuromonitoring. Kaohsiung J Med Sci 26:575-583.

6. Kamani D, Potenza AS, Cernea CR, Kamani YV, Randolph GW 2015 The nonrecurrent laryngeal nerve: anatomic and electrophysiologic algorithm for reliable identification. The Laryngoscope 125:503-508.

7. Chiang FY, Lu IC, Tsai CJ, Hsiao PJ, Lee KW, Wu CW 2012 Detecting and identifying nonrecurrent laryngeal nerve with the application of intraoperative neuromonitoring during thyroid and parathyroid operation. Am J Otolaryngol 33:1-5.

8. Chiang FY, Lu IC, Kuo WR, Lee KW, Chang NC, Wu CW 2008 The mechanism of recurrent laryngeal nerve injury during thyroid surgery--the application of 
intraoperative neuromonitoring. Surgery 143:743-749.

2 9. Snyder SK, Lairmore TC, Hendricks JC, Roberts JW 2008 Elucidating

10. Randolph GW 2013 Surgery of the Thyroid and Parathyroid Glands. Saunders, Elsevier, Philadelphia, PA.

11. Chiang FY, Lee KW, Chen HC, Chen HY, Lu IC, Kuo WR, Hsieh MC, Wu CW 2010 Standardization of Intraoperative Neuromonitoring of Recurrent Laryngeal Nerve in Thyroid Operation. World J Surg 34:223-229.

12. Wu CW, Liu X, Barczynski M, Kim HY, Dionigi G, Sun H, Chiang FY, Kamani D, Randolph GW 2017 Optimal stimulation during monitored thyroid surgery: EMG response characteristics in a porcine model. The Laryngoscope 127:998-1005.

13. Wu CW, Wang MH, Chen CC, Chen HC, Chen HY, Yu JY, Chang PY, Lu IC, Lin YC, Chiang FY 2015 Loss of signal in recurrent nerve neuromonitoring: causes and management. Gland Surg 4:19-26.

14. Wu CW, Hao M, Tian M, Dionigi G, Tufano RP, Kim HY, Jung KY, Liu X, Sun H, Lu IC, Chang PY, Chiang FY 2017 Recurrent laryngeal nerve injury with incomplete loss of electromyography signal during monitored thyroidectomy-evaluation and outcome. Langenbeck's archives of surgery 402:691-699.

15. Lu IC, Chu KS, Tsai CJ, Wu CW, Kuo WR, Chen HY, Lee KW, Chiang FY 2008 Optimal depth of NIM EMG endotracheal tube for intraoperative Neuromonitoring of the recurrent laryngeal nerve during thyroidectomy. World J Surg 32:1935-1939.

16. Tsai CJ, Tseng KY, Wang FY, Lu IC, Wang HM, Wu CW, Chiang HC, Chiang FY 2011 Electromyographic endotracheal tube placement during thyroid surgery in neuromonitoring of recurrent laryngeal nerve. Kaohsiung J Med Sci 27:96-101.

17. Kim HY, Tufano RP, Randolph G, Barczynski M, Wu CW, Chiang FY, Liu X, Masuoka H, Miyauchi A, Park SY, Kwak HY, Lee HY, Dionigi G 2016 Impact of positional changes in neural monitoring endotracheal tube on amplitude and latency of electromyographic response in monitored thyroid surgery: Results from the Porcine Experiment. Head \& neck 38 Suppl 1:E1004-1008.

18. Kartush JM, Lee A 2013 Intraoperative Cranial Nerve Monitoring. In: "Practical Neurotology for the Otolaryngologist". Ed: S Babu. Plural Publishing, Inc. p 165 - 191

19. Barber SR, Liddy W, Kyriazidis N, Cinquepalmi M, Lin BM, Modi R, Patricio S, 
Kamani D, Belotti C, Mahamad S, Lawson B, Randolph GW 2017 Changes in electromyographic amplitudes but not latencies occur with endotracheal tube malpositioning during intraoperative monitoring for thyroid surgery: Implications for guidelines. The Laryngoscope 127:2182-2188.

20. Wu CW, Lu IC, Randolph GW, Kuo WR, Lee KW, Chen CL, Chiang FY 2010 Investigation of optimal intensity and safety of electrical nerve stimulation during intraoperative neuromonitoring of the recurrent laryngeal nerve: a prospective porcine model. Head \& neck 32:1295-1301.

21. Wu CW, Dionigi G, Sun H, Liu X, Kim HY, Hsiao PJ, Tsai KB, Chen HC, Chen HY, Chang PY, Lu IC, Chiang FY 2014 Intraoperative neuromonitoring for the early detection and prevention of RLN traction injury in thyroid surgery: A porcine model. Surgery 155:329-339.

22. Wu CW, Chai YJ, Dionigi G, Chiang FY, Liu X, Sun H, Randolph GW, Tufano RP, Kim HY 2015 Recurrent laryngeal nerve safety parameters of the Harmonic Focus during thyroid surgery: Porcine model using continuous monitoring. The Laryngoscope 125:2838-2845.

23. Riddell V 1970 Thyroidectomy: prevention of bilateral recurrent nerve palsy. Results of identification of the nerve over 23 consecutive years (1946-69) with a description of an additional safety measure. Br J Surg 57:1-11.

24. Chiang FY, Wang LF, Huang YF, Lee KW, Kuo WR 2005 Recurrent laryngeal nerve palsy after thyroidectomy with routine identification of the recurrent laryngeal nerve. Surgery 137:342-347.

25. Dionigi G, Wu CW, Kim HY, Rausei S, Boni L, Chiang FY 2016 Severity of Recurrent Laryngeal Nerve Injuries in Thyroid Surgery. World J Surg 40:1373-1381.

26. Alon EE, Hinni ML 2009 Transcricothyroid electromyographic monitoring of the recurrent laryngeal nerve. The Laryngoscope 119:1918-1921.

27. Petro ML, Schweinfurth JM, Petro AB 2006 Transcricothyroid, intraoperative monitoring of the vagus nerve. Arch Otolaryngol Head Neck Surg 132:624-628.

28. Chiang F-Y, Lu IC, Chang P-Y, Dionigi G, Randolph GW, Sun H, Lee K-D, Tae K, Ji YB, Kim SW, Lee HS, Wu C-W 2017 Comparison of EMG signals recorded by surface electrodes on endotracheal tube and thyroid cartilage during monitored thyroidectomy. The Kaohsiung Journal of Medical Sciences 33:503-509.

29. Puram SV, Chow H, Wu CW, Heaton JT, Kamani D, Gorti G, Chiang FY, Dionigi G, Barczynski M, Schneider R, Dralle H, Lorenz K, Randolph GW 2016 Posterior cricoarytenoid muscle electrophysiologic changes are predictive of vocal cord 
paralysis with recurrent laryngeal nerve compressive injury in a canine model. The Laryngoscope 126:2744-2751.

30

30. Liddy W, Barber SR, Cinquepalmi M, Lin BM, Patricio S, Kyriazidis N, Bellotti C, Kamani D, Mahamad S, Dralle H, Schneider R, Dionigi G, Barczynski M, Wu CW, Chiang FY, Randolph G 2017 The electrophysiology of thyroid surgery: electrophysiologic and muscular responses with stimulation of the vagus nerve, recurrent laryngeal nerve, and external branch of the superior laryngeal nerve. The Laryngoscope 127:764-771. 


\section{Figure Legends}

2

3 Fig 1. Anatomic relationship between the larynx and the surface recording 4 electrodes used for intraoperative neuromonitoring in thyroid surgery.

5 (A). The left and right vocal folds (thyroarytenoid and vocalis muscles) originate from 6 the inner surface of the thyroid cartilage (TC) and extend to the anterior surface of the 7 arytenoid cartilage (AC). The left (circle no.1) and right (circle no. 2) recording 8 electrodes on an endotracheal tube (ETT) are in direct contact with the left and right 9 vocal folds, respectively. Transcartilage recording electrodes (circle no. 3) are 10 attached with medical adhesive to the outer surface of the TC.

11 (B). Commercially available adhesive pre-gelled electrodes (Neotrode II ${ }^{\circledR}$, ConMed 12 Corporation, UTICA, NY) were used for transcartilage EMG recording in this study.

13 (C). A commercially available EMG ETT (TriVantage EMG tube, size 6 ID; 14 Medtronic, Jacksonville, FL). The tube provides bilateral left smooth, conductive 15 silver ink electrodes (left/ right, circle no. $1 / 2$ ) and has a central blue cross-band to 16 guide placement during intubation.

17 (D). Enlarged view of the left (circle no.1) silver ink electrodes on the ETT. 
1 Fig 2. Setup of the endotracheal tube (ETT) and the thyroid cartilage (TC) 2 surface recording electrodes for intraoperative neuromonitoring in a porcine 3 model. (A). The EMG ETT (Medtronic, Jacksonville, FL) was fixed after 4 confirming the correct electrode positions and ensuring good contact with the left and 5 right vocal folds. (B). The animal's neck was exposed with a self-retaining 6 thyroidectomy retractor (WIDOX ${ }^{\circledR}$, MOSS Spa, Lesa, Italy). A pair of adhesive 7 pre-gelled electrodes (Neotrode $\mathrm{II}{ }^{\circledR}$, ConMed Corporation, UTICA, NY) were placed 8 on the left and right lamina of the thyroid cartilage. A Prass monopolar ball-tip 9 stimulator (Medtronic, Jacksonville, FL) was used for nerve stimulation. (C and D). 10 Both the ETT (channel 2. vocal right) and TC (channel 3. TC-1) electrodes 11 successfully recorded typical laryngeal EMG waveforms evoked with $1 \mathrm{~mA}$ stimulus 12 current on the recurrent laryngeal nerve (RLN) (*sign) and vagus nerve (VN) (+ sign). 13 


\section{Fig. 3. Experimental designs for trachea/cricoid displacement and nerve traction}

2 injury studies.

3 (A and B) Tracheal displacement was induced by slowly pushing the tracheal cartilage

4 (T) upward during continuous left vagal stimulation with an automatic periodic 5 stimulating electrode (APS; Medtronic) (* sign). The experiment mimicked

6 tracheal upward displacement caused by retraction of the thyroid lobe that adhered

7 tightly to the trachea by ligament of Berry (LOB) at surgery.

8 (C and D) Recurrent laryngeal nerve (RLN) traction injury was induced by retracting

9 a vascular loop wrapped around the left RLN (R) during continuous left vagal 10 stimulation with an APS electrode (APS) (* sign). The experiment mimics the 11 surgical scenario of an RLN trapped at the ligament of Berry or adhered to the goiter 12 capsule and stretched forward during medial traction. 
1 Fig. 4. Comparison of left and right side evoked laryngeal EMG responses

2 between the endotracheal tube (ETT) and thyroid cartilage (TC) electrodes

3 during intraoperative neuromonitoring. (A) The evoked EMG amplitude

4 (microvolt, $\mu \mathrm{V}$ ) and (B) latency (millisecond, ms) data from left and right recurrent

5 laryngeal nerve (RLN) and vagus nerve (VN) stimulation with $1 \mathrm{~mA}$ were shown and

6 were expressed as mean with standard deviation.

7 
1 Fig. 5. Comparison of real-time electromyography (EMG) signals recorded by 1.

2 Vocalis electrode on endotracheal tube (ETT) and 2. Transcartilage electrode on 3 thyroid cartilage (TC) when tracheal displacement was experimentally induced.

4 The EMG amplitude from the ETT electrodes showed over 50\% decreased (red dot 5 line) when the trachea was displaced over $1 \mathrm{~cm}$. The EMG signals from the TC 6 electrodes showed relatively less affected by the tracheal displacement. 7 
1 Fig. 6. Comparison of real-time electromyography (EMG) signals recorded by 1.

2 Vocalis electrode on endotracheal tube (ETT) and 2. Transcartilage electrode on

3 thyroid cartilage (TC) when recurrent laryngeal nerve (RLN) traction stress was

4 experimentally induced. Both ETT and TC electrodes accurately recorded typical

5 patterns of progressive EMG amplitude degradation during RLN traction with 50gm

6 force. Both electrodes also recorded the typical patterns of gradual EMG amplitude

7 recovery of after release of traction force. 

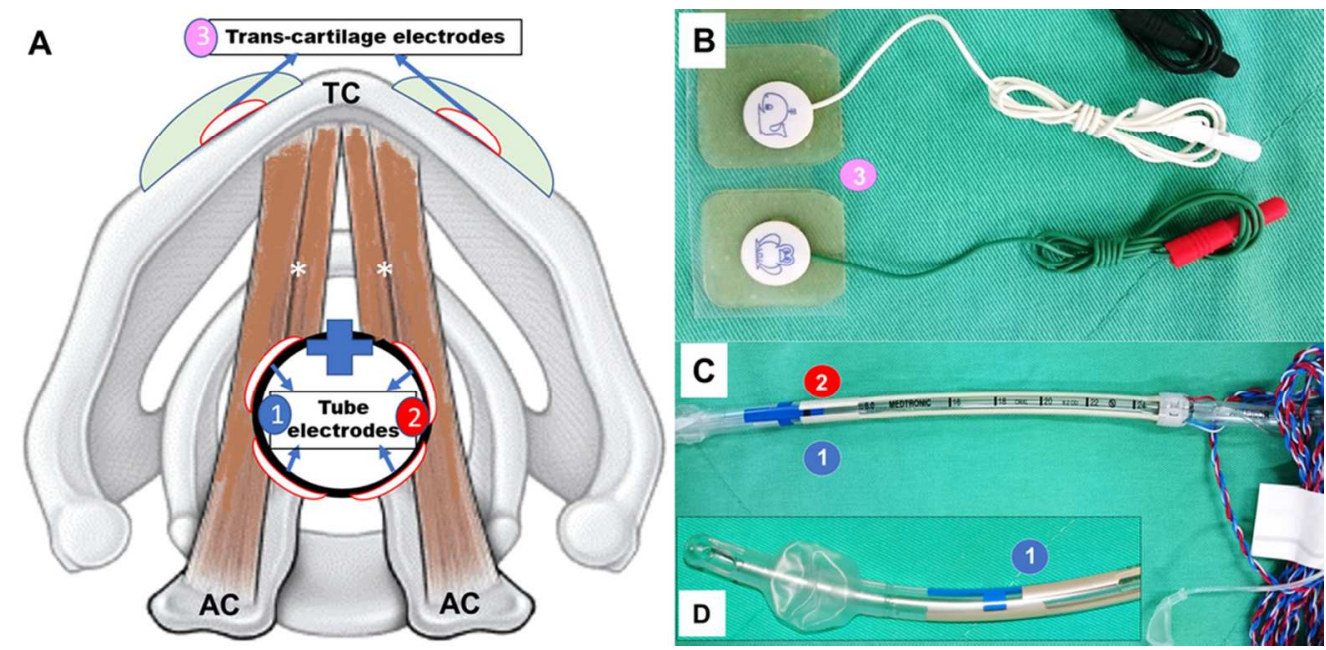

Fig 1. Anatomic relationship between the larynx and the surface recording electrodes used for intraoperative neuromonitoring in thyroid surgery.

(A). The left and right vocal folds (thyroarytenoid and vocalis muscles) originate from the inner surface of the thyroid cartilage (TC) and extend to the anterior surface of the arytenoid cartilage (AC). The left (circle no.1) and right (circle no. 2) recording electrodes on an endotracheal tube (ETT) are in direct contact with the left and right vocal folds, respectively. Transcartilage recording electrodes (circle no. 3) are attached with medical adhesive to the outer surface of the TC.

(B). Commercially available adhesive pre-gelled electrodes (Neotrode II ${ }^{\circledR}$, ConMed Corporation, UTICA, NY) were used for transcartilage EMG recording in this study.

(C). A commercially available EMG ETT (TriVantage EMG tube, size 6 ID; Medtronic, Jacksonville, FL). The tube provides bilateral left smooth, conductive silver ink electrodes (left/ right, circle no. 1/2) and has a central blue cross-band to guide placement during intubation.

(D). Enlarged view of the left (circle no.1) silver ink electrodes on the ETT. 


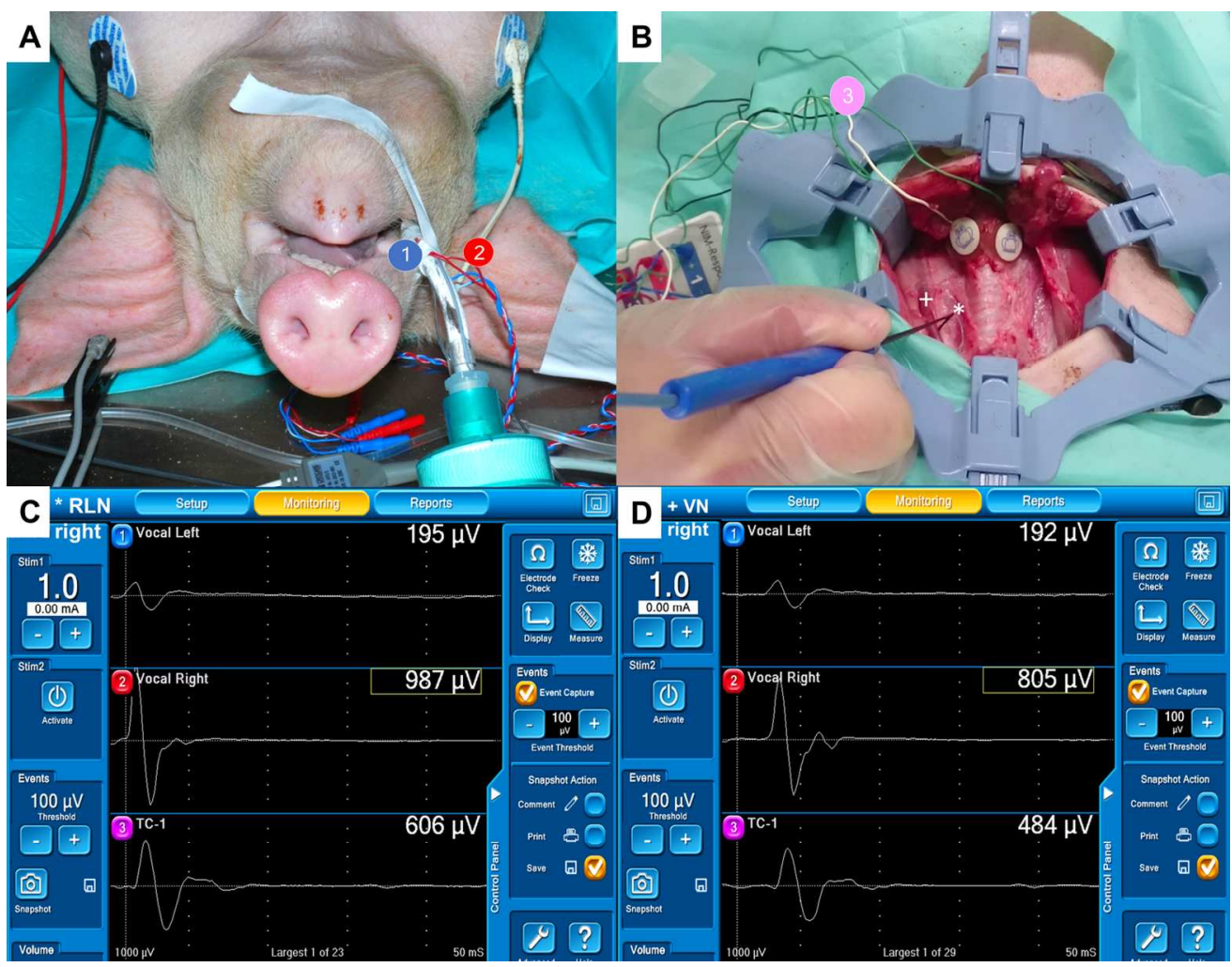

Fig 2. Setup of the endotracheal tube (ETT) and the thyroid cartilage (TC) surface recording electrodes for intraoperative neuromonitoring in a porcine model. (A). The EMG ETT (Medtronic, Jacksonville, FL) was fixed after confirming the correct electrode positions and ensuring good contact with the left and right vocal

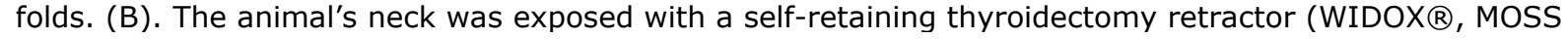
Spa, Lesa, Italy). A pair of adhesive pre-gelled electrodes (Neotrode II ${ }^{\circledR}$, ConMed Corporation, UTICA, NY) were placed on the left and right lamina of the thyroid cartilage. A Prass monopolar ball-tip stimulator (Medtronic, Jacksonville, FL) was used for nerve stimulation. (C and D). Both the ETT (channel 2. vocal right) and TC (channel 3. TC-1) electrodes successfully recorded typical laryngeal EMG waveforms evoked with $1 \mathrm{~mA}$ stimulus current on the recurrent laryngeal nerve (RLN) (*sign) and vagus nerve (VN) (+ sign). 


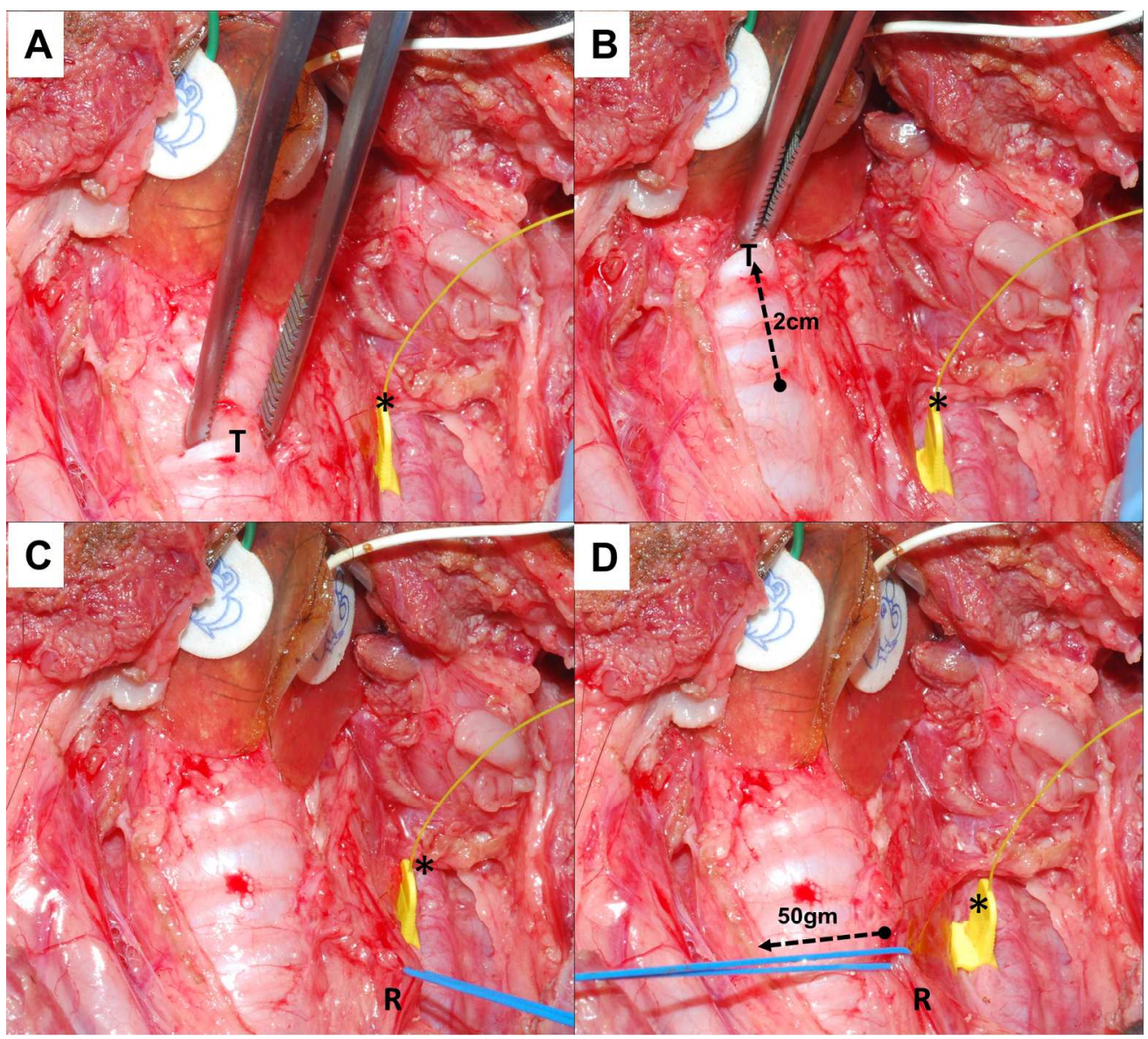

Fig. 3. Experimental designs for trachea/cricoid displacement and nerve traction injury studies.

( $A$ and $B$ ) Tracheal displacement was induced by slowly pushing the tracheal cartilage $(T)$ upward during continuous left vagal stimulation with an automatic periodic stimulating electrode (APS; Medtronic) (* sign). The experiment mimicked tracheal upward displacement caused by retraction of the thyroid lobe that adhered tightly to the trachea by ligament of Berry (LOB) at surgery.

( $C$ and $D$ ) Recurrent laryngeal nerve (RLN) traction injury was induced by retracting a vascular loop wrapped around the left RLN (R) during continuous left vagal stimulation with an APS electrode (APS) (* sign). The experiment mimics the surgical scenario of an RLN trapped at the ligament of Berry or adhered to the goiter capsule and stretched forward during medial traction. 


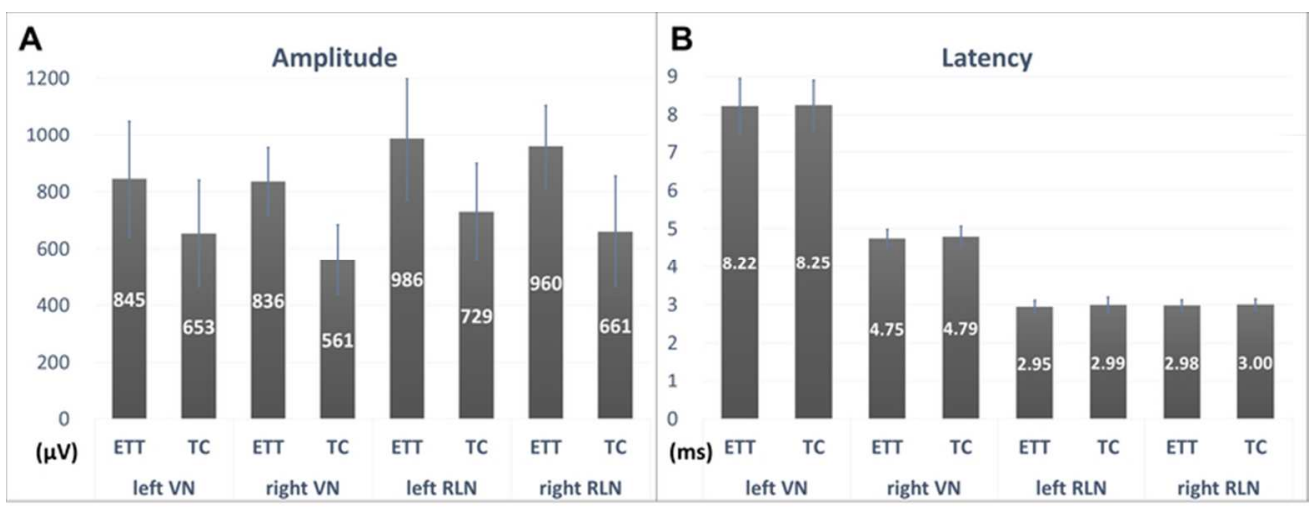

Fig. 4. Comparison of left and right side evoked laryngeal EMG responses between the endotracheal tube (ETT) and thyroid cartilage (TC) electrodes during intraoperative neuromonitoring. (A) The evoked EMG amplitude (microvolt, $\mu \mathrm{V}$ ) and (B) latency (millisecond, ms) data from left and right recurrent laryngeal nerve (RLN) and vagus nerve (VN) stimulation with $1 \mathrm{~mA}$ were shown and were expressed as mean with standard deviation.

$69 \times 26 \mathrm{~mm}(300 \times 300 \mathrm{DPI})$ 


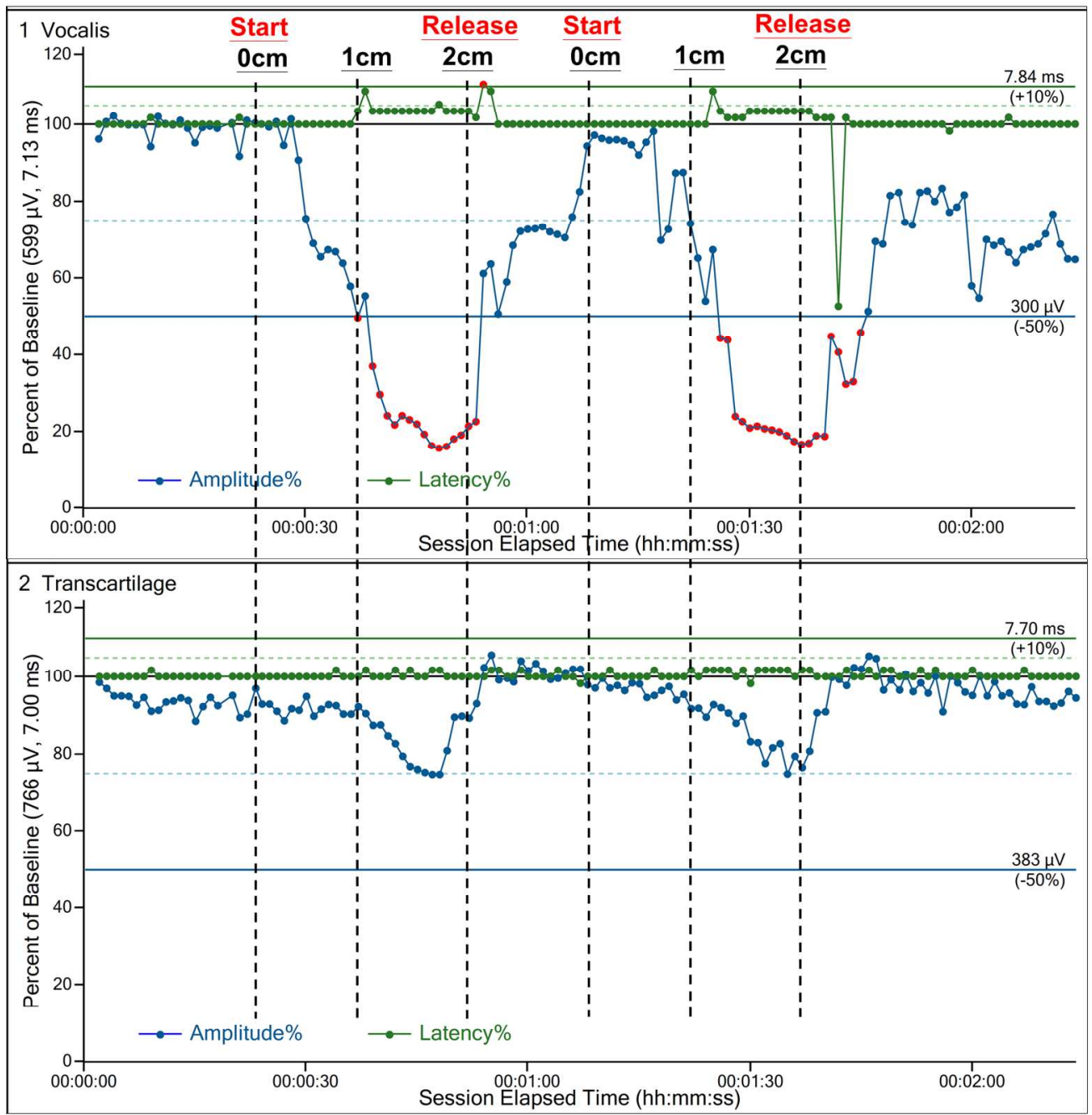

Fig. 5. Comparison of real-time electromyography (EMG) signals recorded by 1 . Vocalis electrode on endotracheal tube (ETT) and 2. Transcartilage electrode on thyroid cartilage (TC) when tracheal displacement was experimentally induced. The EMG amplitude from the ETT electrodes showed over $50 \%$ decreased (red dot line) when the trachea was displaced over $1 \mathrm{~cm}$. The EMG signals from the TC electrodes showed relatively less affected by the tracheal displacement. 


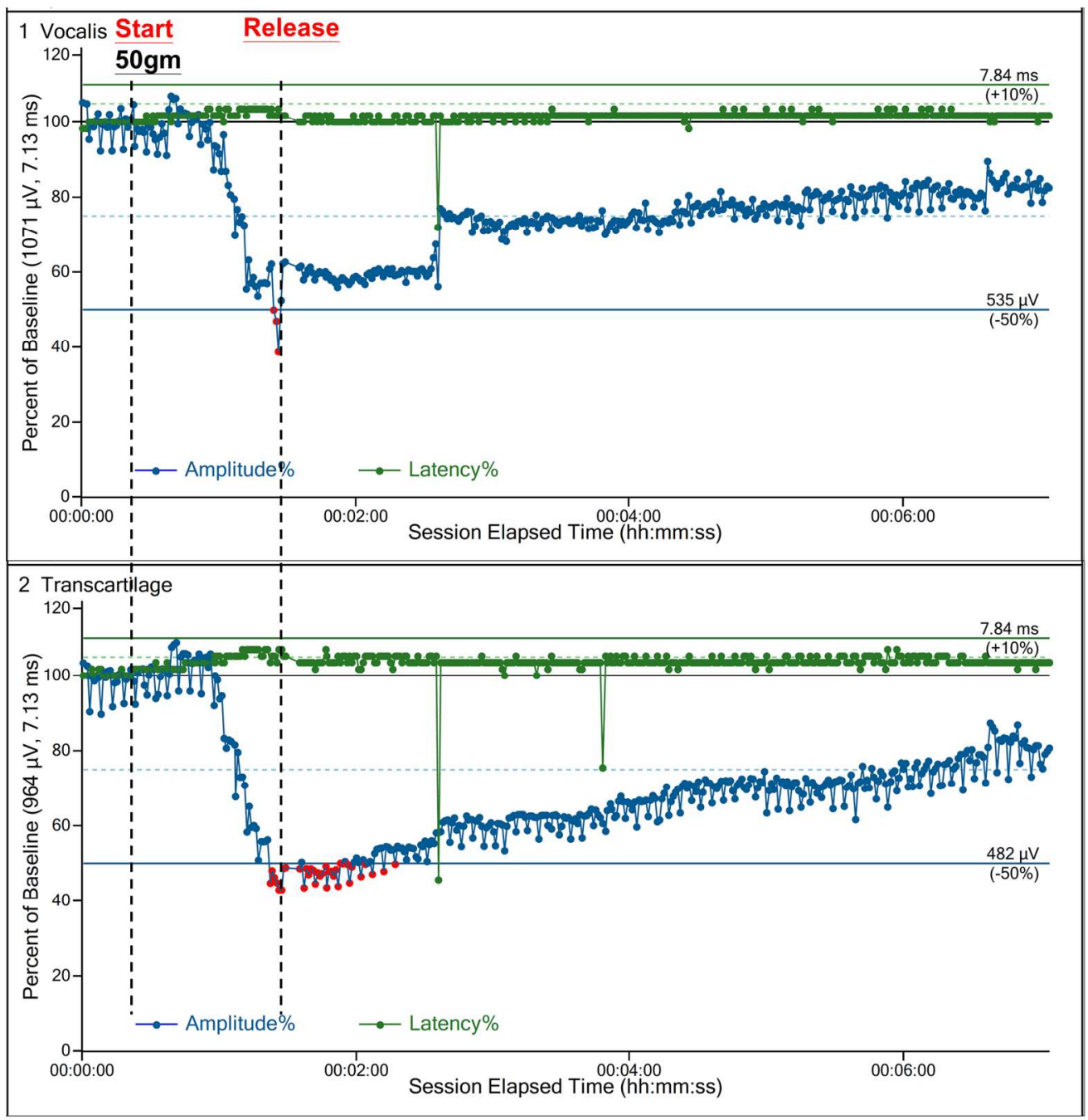

Fig. 6. Comparison of real-time electromyography (EMG) signals recorded by 1 . Vocalis electrode on endotracheal tube (ETT) and 2. Transcartilage electrode on thyroid cartilage (TC) when recurrent laryngeal nerve (RLN) traction stress was experimentally induced. Both ETT and TC electrodes accurately recorded

typical patterns of progressive EMG amplitude degradation during RLN traction with $50 \mathrm{gm}$ force. Both electrodes also recorded the typical patterns of gradual EMG amplitude recovery of after release of traction force. 


\section{$\underline{\text { Cover letter }}$}

Editor, Thyroid

RE: Manuscript ID: THY-2017-0680-R1 entitled, " Feasibility of Intraoperative

Neuromonitoring During Thyroid Surgery using Trans-cartilage Surface Recording

electrodes."

Dear Editors:

Thank you very much for your review and for your excellent advice for improving our manuscript. We have tried our best to incorporate your comments in the resubmitted version, and we agree that the suggested changes make our manuscript clearer and more informative for readers. All important changes are highlighted by using the track changes mode in MS Word in the revised manuscript. With these modifications, we look forward to your further review and consideration of this manuscript for publication in Thyroid.

Sincerely,

Che-Wei Wu, MD, PhD (On behalf of all coauthors)

Department of Otolaryngology - Head and Neck Surgery, Kaohsiung Medical University, Taiwan

Address: 100 TzYou First Road, Kaohsiung City 807, Taiwan

Tel: +886-7-3121101 ext.5009; Fax: +886-7-3208264

E-mail: cwwu@kmu.edu.tw

June, 8, 2018 


\section{Responses to specific comments and questions by the reviewers:}

\section{Reviewer: 1}

\section{Comments to Author}

A commendable article on a novel technique for recording EMG CMAP responses from the larynx. My main comments relate to the applicability of this technology to the human population and to the study design as follows:

1. The are a multitude of muscles that attach to the larynx and underly the surface electrodes (sterno- and thyro-hyoid, inferior constrictor). far field potentials from these muscle could distort the recorded traces and should have been controlled for in this study using hookwire electrodes in these muscles.

2. There are obvious differences between adult human and juvenile pig cartilages. Ossification of the cartilage may significantly impair recording of the CMAP responses and should be commented upon and discussed in the limitations section. 3. There is much discussion in the article regarding ETT limitations. However, the limitations of surface electrodes also need to be discussed including displacement and exposure issues (i.e. need to expose the entire thyroid cartilage which, particularly for minimally invasive approaches, is rarely done). I would also like to know how well these electrodes remain in place during an actual thyroid case - to be stable they would have to stick very firmly and this would surely predispose to bleeding and muscle damage upon removal.

4. The mechanism employed to demonstrate that the electrodes do no more when the ETT does seems to be somewhat biased to show the desired result. Isolated pushing of the cricoid cartilage upward rarely occurs during standard thyroid surgery - instead the vector of force tends to be a combination of rotation and displacement. The rotatory force would likely disrupt the surface electrodes in addition to skewing ETT potentials and this should be acknowledged in the article.

5. The claim that the electrodes can be placed in $<1$ minute needs clarification. Granted sticking an electrode onto a muscle is quick but this does not take into consideration the exposure necessary to do this quick maneuver. Please either remove this sentence or comment that wide exposure is first necessary.

6. The electrodes are prone to displacement during the case which adds a new facet to any LOS troubleshooting algorithm); this should be discussed in limitations 7. Description of time-consuming and cumbersome placement of ETT electrodes should be removed as this is a biased comment (page 14, lines 3-4).

\section{Response:}


Thank you very much for your review and for your excellent advice for improving our manuscript.

1. We agree with your comment that far field potentials from the muscle attached to the larynx could distort the recorded traces. A higher stimulation current may cause shunt stimulation to the ansa cervicalis and/ or the strap muscles, especially during vagal stimulation, and that the evoked EMG could be recorded by surface electrodes. Although these far field potentials can be observed on the monitor screen (different waveforms, especially latencies), they may distort the recorded traces. We have performed additional experiments to clarify how surface electrodes are affected by far field potentials. Supplemental Fig. 1 shows that a very high stimulation current (e.g., 20mA, Supp. Fig. 1B) applied, and electrodes inside (ch1 and ch2, EMG tube electrode) and outside the larynx (ch3, pre-gelled electrode on the skin; ch4, pre-gelled electrode on the thyroid cartilage) detected far field potentials (star sign) from the strap muscles. The effect is most obvious in surface electrodes on the skin (ch3) and on the thyroid cartilage (ch4). In contrast, when the stimulation current is lower than 10mA (e.g., 5mA in Supp. Fig. 1D), far field potentials are too low to distort the recording trace. The standard stimulation current used in clinical practice is between $0.5 \mathrm{~mA}$ and $3 \mathrm{~mA}$ (e.g., $1 \mathrm{~mA}$ in Fig. 2D). Therefore, far field potentials should not substantially affect normal recordings. The issue of far field potentials is addressed in the Discussion section (electrode limitation: No.2.) of the revised manuscript. Again, we appreciate your excellent advice, and we agree that this information makes our manuscript more informative for readers.

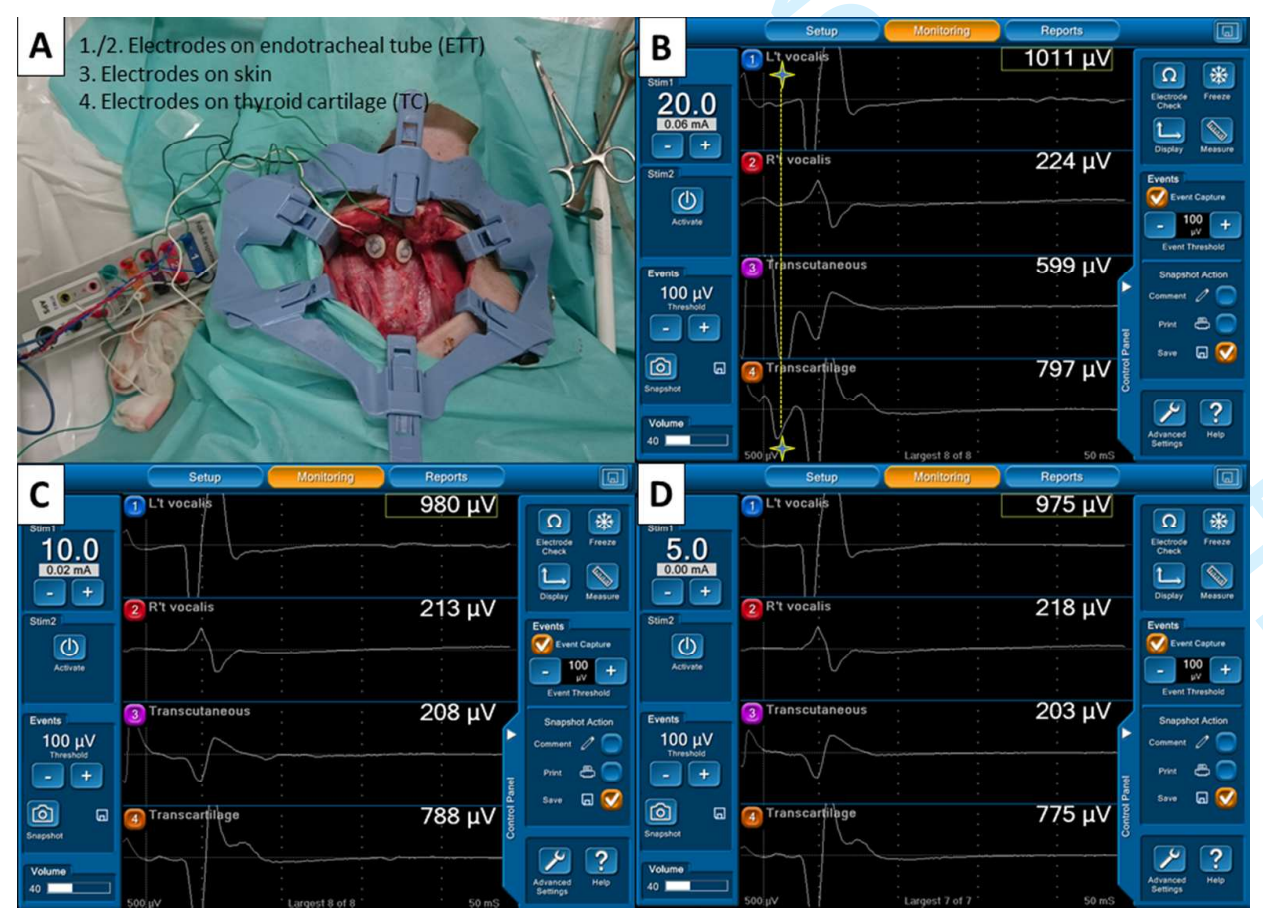


Supp. Fig. 1. Evaluation of far field potentials. (A) Placement of electrodes. (B) EMG data obtained under 20mA stimulation. All electrodes detect far field potentials (star sign) from the strap muscles. (C) EMG data obtained under $10 \mathrm{~mA}$ stimulation. Far field potentials are much decreased. (D) EMG data obtained under $5 \mathrm{~mA}$ stimulation. Far field potentials are very low without distorting the recording trace.

2. Thank you for your comment that ossification may differ between adult human and juvenile pig cartilages. This limitation is noted in the Discussion section of the revised manuscript (animal study limitation: No.2.)

3. Thank you for your insightful observations regarding the limitations of the transcartilage recording method and the adhesive pre-gelled electrodes used in the current study. The following text has been added to the Discussion section of the revised manuscript to address these important limitations. "However, the transcartilage recording method and the adhesive pre-gelled electrodes (Neotrode ${ }^{\circledR}$ II, Fig. 2B) used in the current study have several notable limitations. Firstly, the transcartilage recording method requires a larger incision wound to expose the TC. This method slightly increases surgical time and may not be useful for procedures in which a minimally invasive approach is desirable. ....Secondly, the size and shape of this electrode type are designed for ECG recording on the chest skin of young children. Therefore, these electrode types are somewhat larger than necessary for use on the TC. Recording traces can be distorted by the far field potentials from multitude of muscles attached to the larynx and underlying the surface electrodes (e.g., sterno-hyoid muscle, thyro-hyoid muscle, and inferior constrictor muscle). For example, the experiments in this study showed that a stimulation current exceeding 10 $\mathrm{mA}$ causes some shunt stimulation of the ansa cervicalis and/ or the strap muscles. Evoked muscle potentials could be recorded more easily by large surface electrodes on the TC. However, no obvious far field potentials were recorded under the standard stimulation current of $0.5-3 \mathrm{~mA}$ (Figs. 1C-D). Therefore, new electrode designs with sizes and shapes optimized for TC recording are needed, and further animal and clinical studies are needed to evaluate their susceptibility to far field potentials.

Third, although this study showed that TC electrodes obtained more stable signals than ETT electrodes did during upward displacement of the trachea, the adhesive pre-gelled surface electrodes may still be dislodged during surgical manipulations. Dislodgement can occur because the vector of manipulative force applied during clinical thyroid surgery tends to include both rotatory force and displacement. Rotatory force would likely displace the contact between the ETT electrodes and vocal fold and may also dislodge the TC surface electrodes. ..... To minimize dislodgement, the stability of future electrode designs must be increased by improving 
their adhesion to the TC. Smaller electrodes are also needed to minimize the possibility of damage to cartilage or nearby muscles upon electrode removal. Additionally, the following text has been added to the Conclusion section." A remaining challenge in the practical clinical application of transcartilage IONM is development of surface electrodes that provide a desirable combination of proper size/shape, high adhesive stability, and high sensitivity."

4. Thank you for your important comment on the tracheal displacement study. Significant changes in EMG amplitude with rotation of the endotracheal tube (45/90 degrees) and with changes in depth (upward/downward $1 \mathrm{~cm} \mathrm{\&} 2 \mathrm{~cm}$ ) have been described in animal studies (Head Neck 38: E1004-E1008, 2016) and clinical studies (Laryngoscope, 127:2182-2188, 2017) performed previously by the authors. The aim of this study was to evaluate the feasibility of the transcartilage approach. Only one experiment (induced upward displacement) was performed for comparison of stability and for quantitative analysis of EMG data. We have performed additional experiments in recent months, including an experiment to determine how EMG amplitude change affects rotatory force. The experimental results were consistent, i.e., during medial and lateral rotatory manipulation of the trachea, EMG signal variation was lower in TC electrodes than in ETT electrodes. However, quantitative analysis of the results for the rotatory experiment were very difficult because the true rotatory force vector and the true rotation degree on vocal cord could not be assessed. Since our previous animal experiments did not collect data for the rotatory effect, we only included data for the upward displacement. In the revised version, we also analyze the $1 \mathrm{~cm}$ upward displacement data in addition to the data for $2 \mathrm{~cm}$ upward displacement presented in the original manuscript.

The Discussion section of the revised manuscript notes the important limitation mentioned by the reviewer as follows. " "Third, although this study showed that TC electrodes obtained more stable signals than ETT electrodes did during upward displacement of the trachea, the adhesive pre-gelled surface electrodes may still be dislodged during surgical manipulations. Dislodgement can occur because the vector of manipulative force applied during clinical thyroid surgery tends to include both rotatory force and displacement. Rotatory force would likely displace the contact between the ETT electrodes and vocal fold and may also dislodge the TC surface electrodes."

To avoid bias, material regarding the high stability of the TC electrodes has been deleted from the title, the abstract, and the Results section. 
5. Thank you for this important comment. The revised Discussion section mentions that this approach may require wide exposure: "Firstly, the transcartilage recording method requires a larger incision wound to expose the TC. This method slightly increases surgical time and may not be useful for procedures in which a minimally invasive approach is desirable." The statement that "the electrodes can be placed in $<$ 1 minute" has also been deleted.

6. Thank you. We fully agree with your comment that "the electrodes are prone to displacement during the case which adds a new facet to any LOS troubleshooting algorithm". The following text has been added to the Limitations section.

"Although a dislodged TC electrode can be confirmed by direct visualization and can be corrected immediately, the troubleshooting algorithm for loss of signal must be modified to address this limitation."

7. Thank you for this important comment. The biased statement that ETT electrode placement is time-consuming and cumbersome has been deleted (page 14, lines 3-4). 


\section{Reviewer: 2}

\section{Comments to Author}

The area of research is very relevant to thyroid surgeons globally. The authors aim to improve upon the use of ETT electrodes in IONM and this is considered a standard technique in IONM. The avoidance of ETT electrodes has potential to simplify IONM and reduce costs. The study compares TC to ETT electrodes in a pig model and demonstrates that TC electrodes work well. Although the sensitivity of TC may be lower than ETT electrodes, it appears to be less influenced by movement. In summary, this is a well conducted study, the reporting is clear and concise and this has potential for clinical application after further appropriately conducted human studies are performed.

I have a few queries for the authors to address.

1. Are the TC gel electrodes sterile and are they currently in clinical use? Do they dislodge during surgery and can they be readily replaced?

2. It is not clear if a thyroidectomy was actually performed. If not, why not? It may have been very useful to compare TC and ETT readings during and after thyroidectomy.

3. Could the authors provide some justification for the sample size $(n=10)$ ? 4. No detail is provided on statistical methods. The word 'significant' is used in various places; for eg, to describe changes during tracheal manipulation. Is this statistically significant? What statistical test were used?

5. The last line in the methods section needs to be rewritten to improve clarity.

\section{Response:}

Thank you very much for your review and your encouraging comments on this article.

1. The aim of this experimental study was to test our hypothesis that transcartilage approach is feasible for laryngeal EMG recording during IONM. The intended clinical use of the Neotrode ${ }^{\circledR}$ II adhesive pre-gelled electrodes (Supp. Fig. 2) is ECG recording in young children rather than surgery and thyroidectomy. We agree with your insightful observation that the electrodes could become dislodged during surgery. The Discussion section of the revised manuscript notes this and other limitations of these electrodes as follows." Secondly, the size and shape of this electrode type are designed for ECG recording on the chest skin of young children. Therefore, these electrode types are somewhat larger than necessary for use on the TC.... Therefore, new electrode designs with sizes and shapes optimized for TC recording are needed, and further animal and clinical studies are needed to evaluate their susceptibility to far field potentials... Third, although this study showed that TC electrodes obtained more 
stable signals than ETT electrodes did during upward displacement of the trachea, the adhesive pre-gelled surface electrodes may still be dislodged during surgical manipulations...... Although a dislodged TC electrode can be confirmed by direct visualization and can be corrected immediately, the troubleshooting algorithm for loss of signal must be modified to address this limitation. To minimize dislodgement, the stability of future electrode designs must be increased by improving their adhesion to the TC. Smaller electrodes are also needed to minimize the possibility of damage to cartilage or nearby muscles upon electrode removal.

The following text has also been added to the Conclusion section." A remaining challenge in the practical clinical application of transcartilage IONM is development of surface electrodes that provide a desirable combination of proper size/shape, high adhesive stability, and high sensitivity."

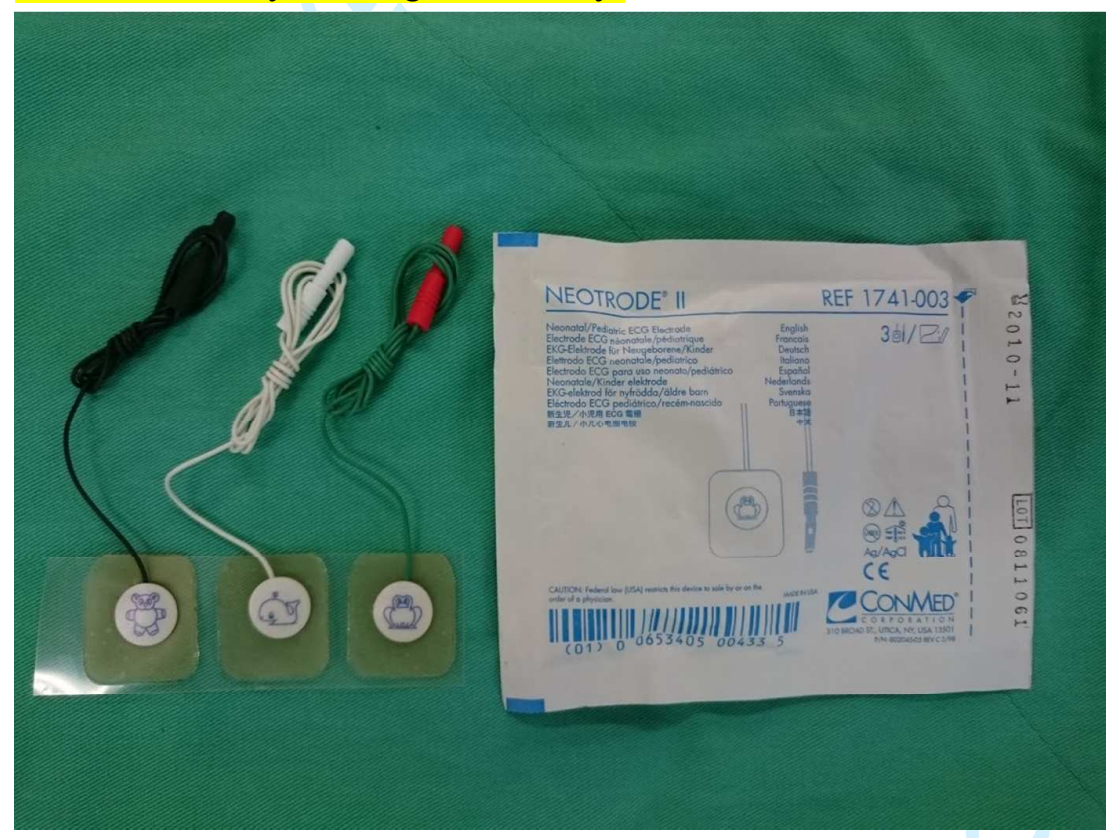

Supp. Fig. 2. Neotrode® II adhesive pre-gelled electrodes.

2. The porcine model used in this study has proven useful for evaluating electrophysiologic correlates of laryngeal EMG during IONM in several studies performed since 2010 (Head Neck. 2010;32(10):1295-301). This model is designed to minimize bias from surgical manipulation by identification of bilateral $\mathrm{VN}$ and RLNs and then dissection to free them from overlying soft tissue (including thyroid tissue) before the electrophysiology EMG experiments are performed (Fig. 2B). After baseline EMG data were documented, surgical manipulations were performed, or RLN injury was experimentally induced to mimic the surgical scenario. The EMG changes were then observed. Therefore, the porcine model used in this study was consistent with the original design. The Materials and Methods section of the revised 
manuscript provides additional clarification and illustration (including a new Fig. 3) of the experimental model.

3. The literature indicate that, since pigs are larger experimental animals than rats, mice, or rabbits, the sample size for most studies that have used pig models ranges from 1 to 16 animals. The study protocol followed the 3R principles (replacement, reduction and refinement) and was approved by the Institutional Animal Care and Use Committee (IACUC approval No.: 105158; totally, 12 experimental animals allowed, Supp. Fig. 3). In the past few months, we have performed two additional animal experiments (therefore, $\mathrm{n}=12$ in the resubmitted manuscript) for further study of the stability and accuracy issues. The experimental results showed good reproducibility. The Discussion section of the revised manuscript notes the limitations regarding the animal model and sample size.

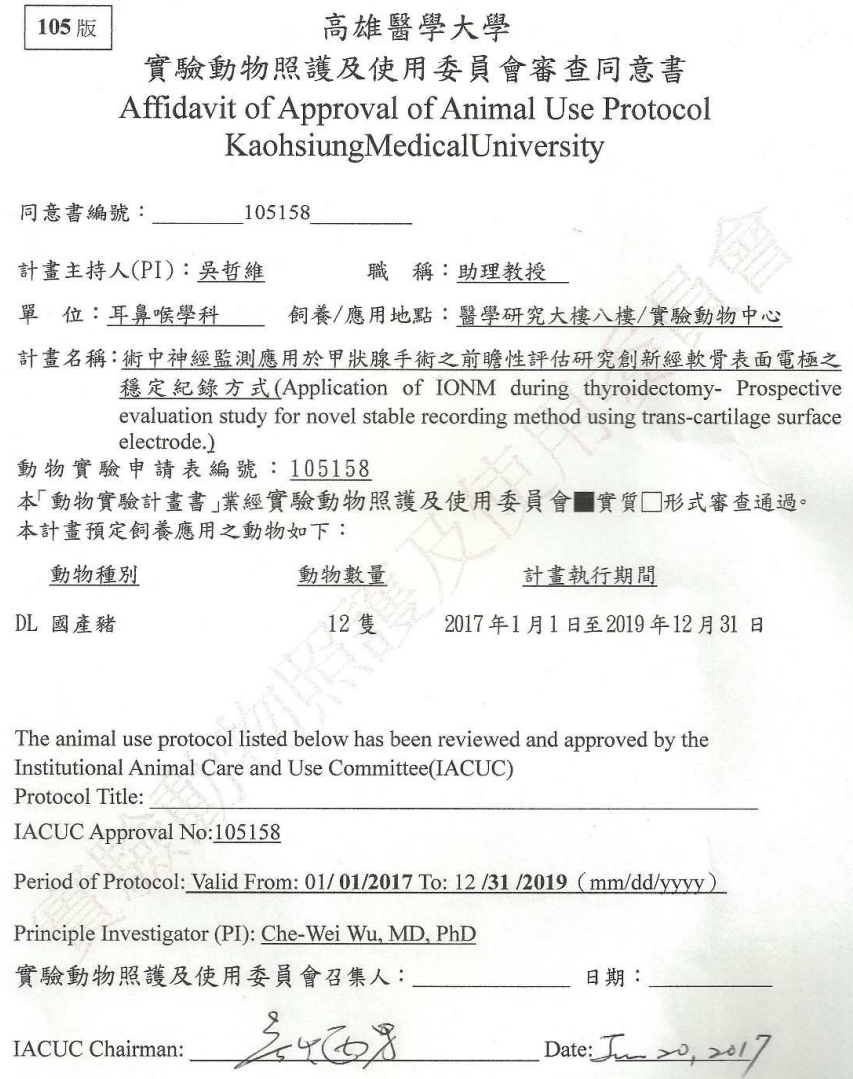

Supp. Fig. 3 Affidavit of Approval of Animal Use Protocol. 
4. Thank you for this important comment. To address this issue, the following text has been added to the "Statistical Analysis" section. "Recorded EMG data were expressed as mean with standard deviation. Mean EMG amplitudes and mean EMG latencies for each stimulation site were compared using paired T-tests. All p-values were two-tailed, and a P value less than 0.05 was considered statistically significant. All statistical analyses were performed using Excel 2016 (Microsoft, Redmond, WA)."

5. Thank you for noting this error. For improved clarity, the text in the last line of the Methods section has been deleted and moved to the Fig. 3 legend. 


\section{Reviewer: 3}

\section{Comments to Author}

The authors present a very interesting alternative to tube electrodes in an animal model. Possibly the authors may enhance on few aspects:

1. Is placement of the EC electrodes possible in lange goiter

2. How are potentials increased to compete with traditonal IONM

3. Changes in amplitude during manipulation are mostly sideways rarely as much as 2 centimeters axonial, thus better models may be tested

4. Comparison of left vs right ampitudes between both types may be elaborated In all a positive perspective to be further investigated

\section{Response:}

Thank you very much for your review and your encouraging comments on this article. 1. Thank you for this important question regarding the electrode placement. The revised Discussion section notes that, in the case of a large goiter extending to the thyroid cartilage, prelaryngeal dissection of the pyramidal lobe is required before placement of TC electrodes.

2. Thank you for your insightful observation that low amplitude is a limitation of TC electrodes. The revised manuscript addresses this limitation and mentions the need for further studies to evaluate new electrode materials and to compare different transduction mechanisms of surface electrodes to improve sensitivity. 3. Thank you for this important comment. Significant changes in EMG amplitude with rotation of the endotracheal tube (45/90 degrees) and with changes in depth (upward/downward displacements of $1 \mathrm{~cm}$ and $2 \mathrm{~cm}$ ) have been reported in previous animal studies (Head Neck 38: E1004-E1008, 2016) and clinical studies (Laryngoscope, 127:2182-2188, 2017) performed by the authors. The aim of this study was to evaluate the feasibility of the transcartilage approach, and only one experiment (induced upward displacement) was performed for comparison of stability and for quantitative analysis of EMG data. We have recently performed additional experiments, including an experiment to test how rotatory force affects EMG amplitude. The experimental results were consistent, i.e., variation was lower in EMG signals detected by TC electrodes than in those detected by ETT electrodes during medial and lateral rotatory manipulation of the trachea. However, quantitative analysis of the results for the rotatory experiment were very difficult because the true rotatory force vector and the true rotation degree on vocal cord could not be assessed. Since our previous animal experiments did not collect data for the rotatory effect, we only included data for the upward displacement. In the revised version, we also 
analyze the $1 \mathrm{~cm}$ upward displacement data in addition to the data for $2 \mathrm{~cm}$ upward displacement presented in the original manuscript. The following text has been added to the Results section. "The mean changes in EMG amplitude recorded after upward displacement of $1 \mathrm{~cm}$ were $7.8 \pm 1.7 \%$ (range, 5 to $11 \%$ ) for ETT electrodes and $45.6 \pm 8.2 \%$ (range, 28 to $53 \%$ ) for TC electrodes. The mean changes in EMG amplitude recorded after upward displacement of $2 \mathrm{~cm}$ were $14.3 \pm 4.6 \%$ (range, 8 to $25 \%$ ) for ETT electrodes and $68.4 \pm 10.6 \%$ (range, 53 to $85 \%$ ) for TC electrodes." Since tracheal displacement approximating $1 \mathrm{~cm}$ is representative of the typical surgical scenario, data for $1 \mathrm{~cm}$ displacement are more useful than data for $2 \mathrm{~cm}$ displacement.

4. Thank you for this important comment. Figure 4 of the revised manuscript compares left vs right amplitudes between the two electrode types. We believe this modification make our manuscript more informative for the readers. 


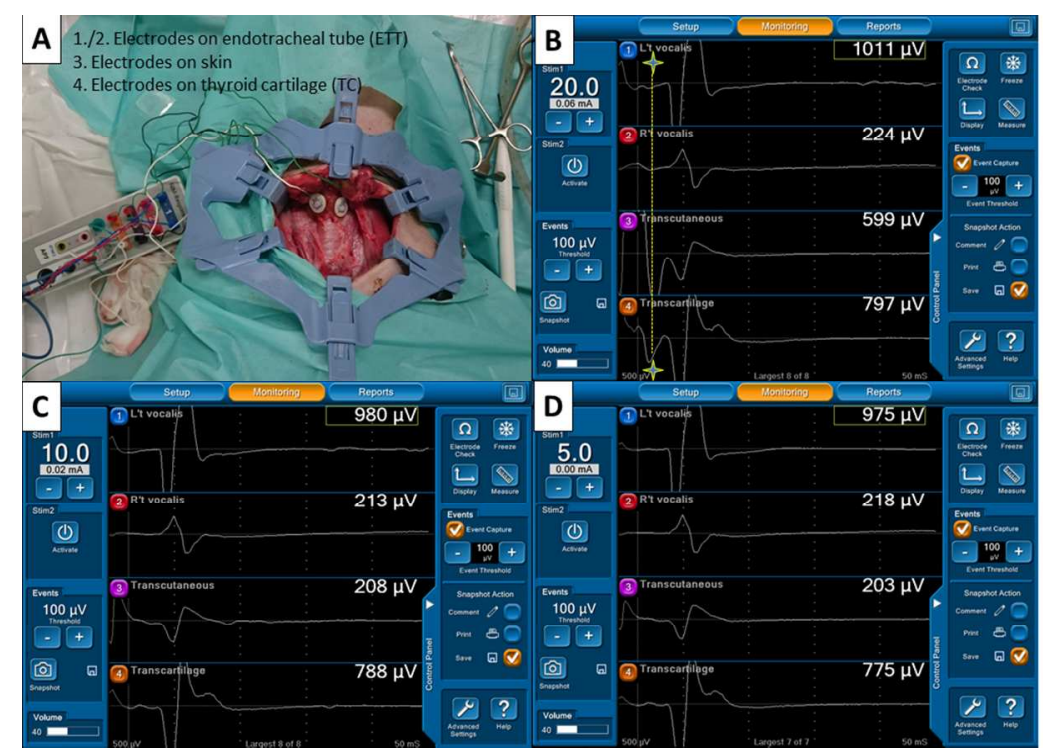

Supp. Fig. 1. Evaluation of far field potentials. (A) Placement of electrodes. (B) EMG data obtained under $20 \mathrm{~mA}$ stimulation. All electrodes detect far field potentials (star sign) from the strap muscles. (C) EMG data obtained under $10 \mathrm{~mA}$ stimulation. Far field potentials are much decreased. (D) EMG data obtained under $5 \mathrm{~mA}$ stimulation. Far field potentials are very low without distorting the recording trace. 


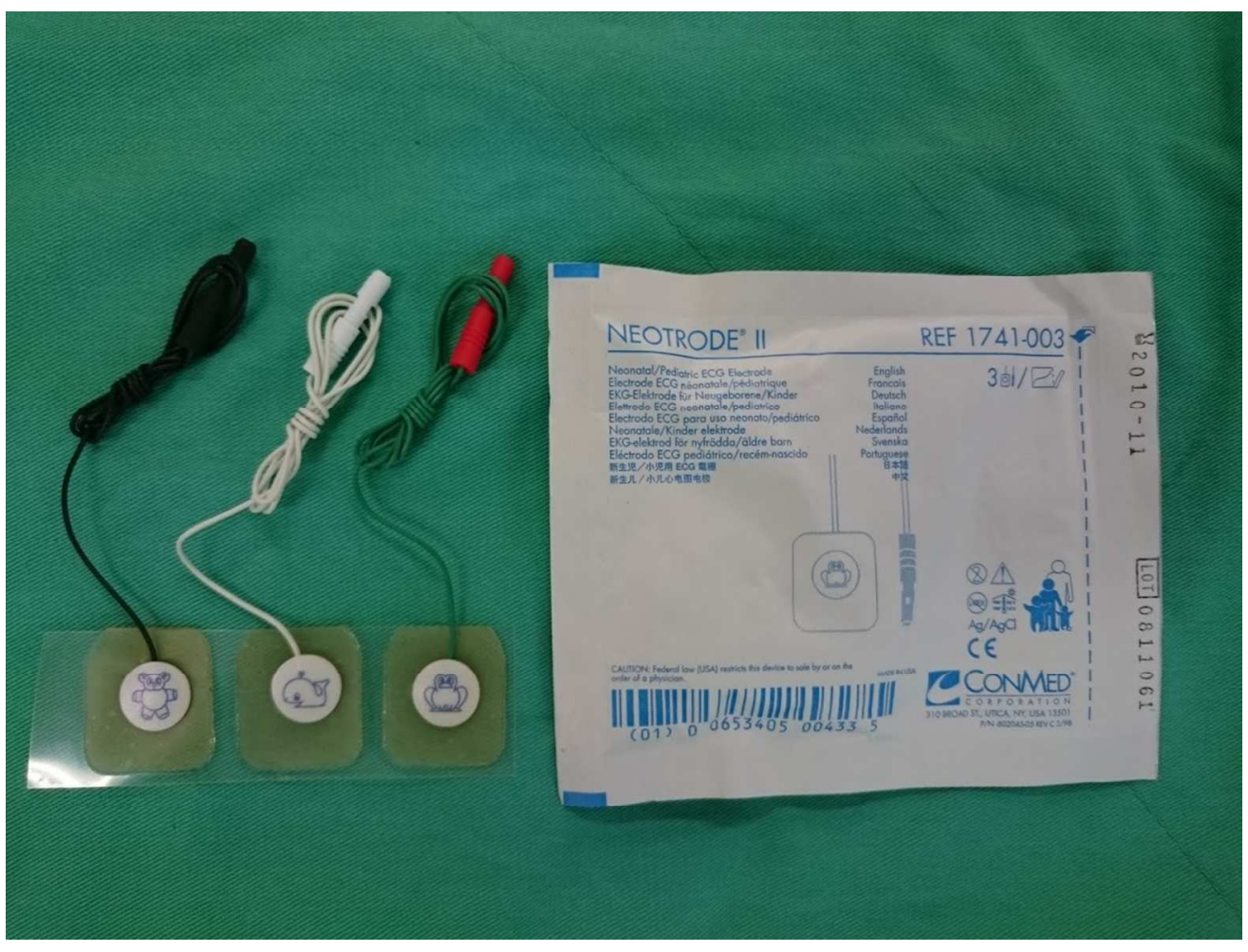

Supp. Fig. 2. Neotrode $®$ II adhesive pre-gelled electrodes.

$458 \times 343 \mathrm{~mm}(72 \times 72 \mathrm{DPI})$ 
105 版高雄醫學大學

實驗動物照護及使用委員會審查同意書 Affidavit of Approval of Animal Use Protocol KaohsiungMedicalUniversity

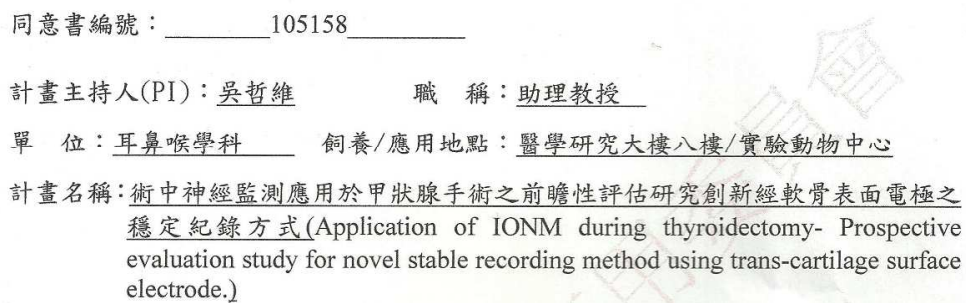

DL 國產橎

2017 年1月 1 日至 2019 年 12 月 31 日

The animal use protocol listed below has been reviewed and approved by the Institutional Animal Care and Use Committee(IACUC) Protocol Title:

IACUC Approval No: 105158

Period of Protocol: Valid From: 01/ 01/2017 To: $12 / 31 / 2019(\mathrm{~mm} / \mathrm{dd} / \mathrm{yyyy})$ Principle Investigator (PI): Che-Wei Wu, MD, PhD

實驗動物照護及使用委員會召集人: 日期：

IACUC Chairman: $\sum_{5}^{8} 4(t)$ Date: In $>0,2017$

Supp. Fig. 3 Affidavit of Approval of Animal Use Protocol. $215 \times 297 \mathrm{~mm}(300 \times 300 \mathrm{DPI})$ 